\title{
The gravitational wave signal from diverse populations of double white dwarf binaries in the Galaxy
}

\author{
S. Yu and C. S. Jeffery
}

Armagh Observatory, College Hill, Armagh, BT61 9DG, Northern Ireland, UK

e-mail: syu@arm.ac.uk, csj@arm.ac.uk

Received 20 April 2010 / Accepted 19 July 2010

\section{ABSTRACT}

\begin{abstract}
Context. The gravitational wave (GW) background in the range $0.01-30 \mathrm{mHz}$ has been assumed to be dominated by unresolved radiation from double white dwarf binaries (DWDs). Recent investigations indicate that, at short periods, a number of DWDs should be resolvable sources of GW.

Aims. We characterize the GW signal which would be detected by LISA from DWDs in the Galaxy.

Methods. We have constructed a Galactic model in which we consider distinct contributions from the bulge, thin disc, thick disc, and halo, and subsequently executed a population synthesis approach to determine the birth rates, numbers, and period distributions of DWDs within each component.

Results. In the Galaxy as a whole, our model predicts the current birth rate of DWDs to be $3.21 \times 10^{-2} \mathrm{yr}^{-1}$, the local density to be $2.2 \times 10^{-4} \mathrm{pc}^{-3}$ and the total number to be $2.76 \times 10^{8}$. Assuming SNIa are formed from the merger of two CO white dwarfs, the SNIa rate should be $0.0013 \mathrm{yr}^{-1}$. The frequency spectra of DWD strain amplitude and number distribution are presented as a function of galactic component, DWD type, formation channel, and metallicity.

Conclusions. We confirm that $\mathrm{CO}+\mathrm{He}$ and $\mathrm{He}+\mathrm{He}$ white dwarf (WD) pairs should dominate the GW signal at very high frequencies $\left(\log f \mathrm{~Hz}^{-1}>-2.3\right)$, while $\mathrm{CO}+\mathrm{CO}$ and $\mathrm{ONeMg}$ WD pairs have a dominant contribution at $\log f \mathrm{~Hz}^{-1} \leq-2.3$. Formation channels involving two common-envelope (CE) phases or a stable Roche lobe overflow phase followed by a CE phase dominate the production of DWDs detectable by LISA at $\log f \mathrm{~Hz}^{-1}>-4.5$. DWDs with the shortest orbital periods will come from the CE+CE channel. The Exposed Core plus CE channel is a minor channel. A number of resolved DWDs would be detected, making up 0.012\% of the total number of DWDs in the Galaxy. The majority of these would be $\mathrm{CO}+\mathrm{He}$ and $\mathrm{He}+\mathrm{He}$ pairs formed through the $\mathrm{CE}+\mathrm{CE}$ channel.
\end{abstract}

Key words. gravitational waves - binaries: close - white dwarfs - Galaxy: structure - galaxies: stellar content

\section{Introduction}

Substantial efforts have been made to measure gravitational waves that, according to Einstein, should permeate the whole Universe (Press \& Thorne 1972; Tyson \& Giffard 1978). Today, four major ground-based detectors are in operation (LIGO, VIRGO, GEO600, and TAMA300), but no direct evidence for the existence of gravitational waves (GW) has been established.

Following the proposed launch of LISA (Laser Interferometer Space Antennae), it is anticipated that the most easily detected waves will originate from close compact binary stars. In addition to providing a direct test of general relativity, the observations of GW from such systems will contribute to understanding close binary star evolution, the distribution of X-ray sources, $\gamma$-ray bursts and supernovae explosions, and may also improve knowledge of galactic structure.

As a result of recent and current surveys, increasing numbers of candidate compact binaries are being found, including double neutron stars (NS) and double white-dwarf (DWD) systems. AM CVn variables are thought to be semi-detached ultracompact binary stars systems in which a white dwarf accretes from a degenerate (or semi-degenerate) companion with an orbital period of less than 80 min. Currently the number of known AM CVn systems is approximately 20 (Ramsay et al. 2007; Roelofs et al. 2007), while those of detached DWD and NS systems with known orbital periods are 24 and 8 respectively.
Theoretical studies of the dominant GW signal in the Galaxy have been pursued for several decades. Mironovskii (1966) calculated the spectral density and total flux due to GW from W UMa binaries and predicted a very low flux with a spectrum peaked at a period of about $0.17 \mathrm{~d}(0.07 \mathrm{mHz})(\mathrm{A}-$ and W-type W UMa binaries have orbital periods in the range $0.4-0.8 \mathrm{~d}$ and $0.22-0.4 \mathrm{~d}$, respectively). Subsequently Evans et al. (1987) demonstrated that degenerate dwarf binaries should be detectable sources of gravitational waves. More recent work indicates that DWD binaries are crucial GW sources in the 0.1 $-100 \mathrm{mHz}$ range (Hils et al. 1990; Hils \& Bender 2000; Nelemans et al. 2001b, 2004; Farmer \& Phinney 2003; Willems et al. 2007). The results of Ruiter et al. (2009) show the halo signal would have a negligible effect on the detection of LISA sources in comparison with that of the disc and bulge.

Such population synthesis models are sensitive to the adopted inputs, including the fast stellar evolution models, the Galactic model, including mass and metallicity distribution, initial mass function, and star formation rate, and various parameters governing binary star evolution, such as mass loss and exchange in binary stars. It is important to ensure that previous results are robust by reproducing them in independent calculations, and to identify the major areas of uncertainty in these models. It is also important to validate a new code by testing it against established benchmarks, before using it to explore new physics. This paper addresses both of these functions. 
To discuss the GW background due to a population of galactic DWDs, we have executed a simulation based on a population synthesis approach for DD binaries which uses a multicomponent model of the Galaxy. We do not include the contribution of DD's containing an NS or black hole (BH) since the formation and evolution of these await further theoretical and observational constraint. Other parameters of our stellar evolution model are taken from the best model of Han (1998).

In addition to the GW signal, our model provides an estimate of the current birth rate, total numbers and properties (e.g. the primary mass and orbital period) of DWDs which may be compared with observation.

In Sect. 2 we describe the model for generating a GW signal from a binary, the stellar evolution model for generating a population of compact binaries, and the model for generating their galactic distribution. In Sect. 3, we show the main results from these models in terms of the overall Galactic GW signal, and in terms of the contribution from various components of the DWD population. Section 4 compares these results with previous work, and conclusions are drawn in Sect. 5.

\section{Simulations}

\subsection{The GW signal from a binary star}

The power of the GW radiated from two point masses in the $n$th harmonic is given by

$L_{\mathrm{GW}}(n, e)=\frac{32}{5}\left(\frac{G^{4}}{c^{5}} \frac{\mu^{2} M^{3}}{a^{5}}\right) g(n, e)$

where $G$ is Newton's gravitational constant, $c$ is the speed of light, $a$ is their orbital separation, $e$ is the eccentricity of the orbit, $M$ and $\mu$ are the total and reduced masses of the system, and $g(n, e)$ is a coefficient expressing the relative power in each harmonic and is given by Peters \& Mathews (1963). If $m_{1}$ and $m_{2}$ are the masses of the components, then

$M=m_{1}+m_{2}, \quad \mu=m_{1} m_{2} / M$.

The GW energy flux density incident on the Earth is

$\frac{L_{\mathrm{GW}}}{4 \pi d^{2}}=\frac{c^{3}}{16 \pi G}\left\langle\dot{h}_{+}^{2}+\dot{h}_{\times}^{2}\right\rangle$,

where $d$ is the distance, $h_{+}$and $h_{\times}$are dimensionless functions for the amplitude of the wave in two orthogonal polarizations, and dots denote time derivatives (Isaacson 1968; Press \& Thorne 1972; Evans et al. 1987). Assuming a binary system in a circular orbit of period $P_{\text {orb }}$ at a distance $d, h_{+}$and $h_{\times}$are given by

$h_{+}=\frac{G^{5 / 3}}{c^{4}} \frac{1}{d} 2\left(1+\cos ^{2} i\right)\left(\pi f_{\mathrm{GW}} M\right)^{2 / 3} \mu \cos \left(2 \pi f_{\mathrm{GW}} t\right)$,

$h_{\times}= \pm \frac{G^{5 / 3}}{c^{4}} \frac{1}{d} 4 \cos i\left(\pi f_{\mathrm{GW}} M\right)^{2 / 3} \mu \sin \left(2 \pi f_{\mathrm{GW}} t\right)$,

where $f_{\mathrm{GW}}=2 / P_{\text {orb }}$ is the frequency of the emitted GW, and $i$ is the inclination angle $\left(i=90^{\circ}\right.$ indicates a binary which is viewed edge-on) (Landau \& Lifshitz 1975).

The so-called strain amplitude $h$, defined as $h^{2}=\frac{1}{2}\left[h_{+, \max }^{2}+\right.$ $\left.h_{\times, \max }^{2}\right]$, describes the strength of the signal to be measured by $\mathrm{GW}$ detectors. From Eqs. (3)-(5), we obtain

$h=\left(\frac{4 G L_{\mathrm{GW}}}{\pi c^{3} f_{\mathrm{GW}}^{2} 4 \pi d^{2}}\right)^{1 / 2}$
(Douglass \& Braginsky 1979; Nelemans et al. 2001b). So from Eqs. (1), (6), and Kepler's law $a^{3} / P_{\text {orb }}^{2}=G\left(m_{1}+m_{2}\right) / 4 \pi^{2}$, we can write $h(n, e)$ in the $n$th harmonic as

$$
\begin{aligned}
h(n, e)= & 1.0 \times 10^{-21} \\
& \times\left(\frac{g(n, e)}{n^{2}}\right)^{1 / 2}\left(\frac{\mathcal{M}}{M_{\odot}}\right)^{5 / 3}\left(\frac{P_{\text {orb }}}{\mathrm{h}}\right)^{-2 / 3}\left(\frac{d}{\mathrm{kpc}}\right)^{-1}
\end{aligned}
$$

Equation (7) gives the strain amplitude in the $n$th harmonic with a frequency $f_{\mathrm{GW}}^{n}=n / P_{\mathrm{orb}}$, and where $\mathcal{M} \equiv \mu^{3 / 5} M^{2 / 5}$ is the socalled chirp mass. Especially for a circular orbit, i.e. $e=0, n=$ 2, we have

$$
\begin{aligned}
h(n, e)= & h(2,0)=5.0 \times 10^{-22} \\
& \times\left(\frac{\mathcal{M}}{M_{\odot}}\right)^{5 / 3}\left(\frac{P_{\text {orb }}}{\mathrm{h}}\right)^{-2 / 3}\left(\frac{d}{\mathrm{kpc}}\right)^{-1}
\end{aligned}
$$

\subsection{Stellar evolution models}

A binary star population synthesis calculation requires the predicted evolution of many thousands of binary stars, only a fraction of which yield systems of interest (Sect. 2.2.6). We have obtained this predicted evolution using the fast binary star evolution (BSE) code described by Hurley et al. $(2000,2002)$ and previously referred to by Han (1998). However, we have modified the code in a number of ways, including several of the approximation formulae and the treatment of the common envelope ejection mechanism. This section describes these changes, including the approximations used to simulate changes in mass and angular momentum due to the interaction of binary star components.

\subsubsection{Common-envelope (CE) evolution}

RLOF occurs in binary systems when one star fills its Roche lobe either by evolutionary expansion or by orbital shrinkage. The Roche radius of the primary is given by

$$
\frac{R_{\mathrm{L}_{1,2}}}{a}=\frac{0.49 q_{1,2}^{2 / 3}}{0.6 q_{1,2}^{2 / 3}+\ln \left(1+q_{1,2}^{1 / 3}\right)}
$$

where $a$ is more generally the semi-major axis of the orbit and $q_{1,2}=m_{1,2} / m_{2,1}$ (Eggleton 1983). Studies of the critical value for stable RLOF have shown that $q_{\mathrm{c}}$ is a function of primary mass $m_{1}$, its core mass $m_{1 \mathrm{c}}$, and mass-transfer efficiency of the donor. We adopt

$q_{\mathrm{c}}=\left(1.67-x+2\left(\frac{m_{1 \mathrm{c}}}{m_{1}}\right)^{5}\right) / 2.13$

where $x=0.3$ is the exponent of the mass-radius relation at constant luminosity for giant stars (Hurley et al. 2000, 2002).

The calculation of the properties (e.g. orbital separation) of a binary after $\mathrm{CE}$ ejection in our model is based on the variation of angular momentum ( $\gamma$-mechanism). In the case of nonconservative mass transfer, the angular momentum loss of a binary system would be described by the decrease of primary mass times a $\gamma$ factor (Paczyński \& Ziółkowski 1967; Nelemans et al. 2000):

$\frac{J_{\mathrm{i}}-J_{\mathrm{f}}}{J_{\mathrm{i}}}=\gamma \frac{m_{1}-m_{1 \mathrm{c}}}{M}$,

where $J_{\mathrm{i}}$ is the orbital angular momentum of the pre-mass transfer binary, and $J_{\mathrm{f}}$ is the final orbital angular momentum after 
CE ejection. If initial and final orbits are assumed circular, the fraction of angular momentum lost during the mass transfer, $J_{\mathrm{i}}-J_{\mathrm{f}}$, becomes

$J_{\mathrm{i}}-J_{\mathrm{f}} \approx \frac{m_{1} m_{2}}{M} a_{\mathrm{i}}^{2} \omega_{\mathrm{i}}-\frac{m_{1 \mathrm{c}} m_{2}}{\left(m_{1 \mathrm{c}}+m_{2}\right)} a_{\mathrm{f}}^{2} \omega_{\mathrm{f}}$

where $\omega_{\mathrm{i}}$ and $\omega_{\mathrm{f}}$ are the circular frequency of the binary before and after CE ejection. Combining (11) and (12) with Kepler's law, we have the ratio of final to initial orbital separation

$\frac{a_{\mathrm{f}}}{a_{\mathrm{i}}}=\left(\frac{m_{1}}{m_{1 \mathrm{c}}}\right)^{2}\left(\frac{m_{1 \mathrm{c}}+m_{2}}{M}\right)\left(1-\gamma \frac{m_{1}-m_{1 \mathrm{c}}}{M}\right)^{2}$.

Nelemans \& Tout (2005) investigated the mass-transfer phase of the progenitors of white dwarfs in binaries employing the $\gamma$-mechanism based on 10 observed systems and suggested the value of $\gamma$ in the range of $1.4 \sim 1.7$. We here adopt $\gamma=1.5$, following the recommendation by Nelemans et al. (2000).

In order to eject a binary star common envelope, conservation of energy requires that the envelope binding energy, including gravitational binding and recombination energies, must be equal to the orbital energy (Webbink 1984, 2008). The ratio of final to initial orbital separation can be expressed as

$$
\begin{aligned}
\frac{a_{\mathrm{f}}}{a_{\mathrm{i}}}= & \frac{m_{1 \mathrm{c}}}{m_{1}}\left[1+2\left(\frac{1}{\beta_{\mathrm{CE}} \lambda_{\Omega} R_{\mathrm{L} 1}}-\frac{1}{\lambda_{\mathrm{P}} R_{L 1}}-\frac{\chi_{\mathrm{eff}} a_{\mathrm{i}}}{G m_{1}}\right)\right. \\
& \left.\times\left(\frac{m_{1}-m_{1 \mathrm{c}}}{m_{2}}\right)\right]^{-1},
\end{aligned}
$$

where the coefficients are given by Webbink (2008).

Both CE ejection mechanisms can reproduce observations (Nelemans et al. 2000; Nelemans \& Tout 2005; Webbink 2008). We here adopt the $\gamma$-algorithm in order to reduce the number of free parameters in the model.

We note that there is a major difference between the $\alpha$ - and $\gamma$-algorithms. In both, the first CE phase can lead to the production of a low-mass binary with $q \approx 1$. However, the $\alpha$-algorithm requires a significant spiral-in stage in order to eject the envelope whilst the $\gamma$-mechanism does not, implying that the orbital separation can be larger after $\mathrm{CE}$ ejection in the latter case (Nelemans et al. 2000; Webbink 2008).

\subsubsection{Stellar wind and accretion}

We assume that the existence of a close companion will increase the rate of mass-loss $(\dot{m})$ due to a stellar wind from a star in a binary system. The tidal enhancement of $\dot{m}_{1}$ is modelled by Reimers (1975) formula with an extra tidal term by Tout \& Eggleton (1988):

$\dot{m}_{1}=-4 \times 10^{-13} \frac{r_{1} l_{1}}{m_{1}}\left(1+B \times\left(\min \left(\frac{r_{1}}{r_{\mathrm{L}_{1}}}, \frac{1}{2}\right)\right)^{6}\right) \mathrm{yr}^{-1}$,

where $r_{1}, l_{1}$ and $m_{1}$ are stellar radius, luminosity and mass in solar units, respectively. We take $B=1000$ (Han 1998).

Some of the mass lost in the wind may be accreted by the companion. The classical accretion rate formula is given by Bondi \& Hoyle (1944), and the mean accretion rate (Boffin \& Jorissen 1988) is

$\dot{m}_{2}=\frac{-1}{\sqrt{1-e^{2}}}\left(\frac{G m_{2}}{v_{\mathrm{w}}^{2}}\right)^{2} \frac{\beta_{\mathrm{w}}}{2 a^{2}} \frac{1}{\left(1+v^{2}\right)^{3 / 2}} \dot{m}_{1}$, where $1 \leqslant \beta_{\mathrm{w}} \leqslant 2$ is an arbitrary parameter $\left(\beta_{\mathrm{w}}=3 / 2\right.$, Boffin et al. 1993), $v_{\mathrm{w}}$ is the wind velocity, $v^{2}=v_{\mathrm{orb}}^{2} / v_{\mathrm{w}}^{2}$, and $v_{\mathrm{orb}}=$ $(G M / a)^{1 / 2}$ is the orbital velocity. Here, we adopt $v_{\mathrm{w}}=20 \mathrm{~km} \mathrm{~s}^{-1}$. The angular momentum of a star will change if the star loses or gains mass. We assume that the specific angular momentum in the wind is proportional to the angular velocity at the surface of the mass-losing star, and that the wind angular momentum is transferred with $100 \%$ efficiency (Hurley et al. 2002).

\subsubsection{Gravitational radiation and magnetic braking}

A close compact binary system driven by gravitational radiation would eventually undergo a mass transfer phase, ultimately leading to coalescence. The orbital changes due to gravitational radiation from two point masses are predicted to be (Hurley et al. 2002):

$$
\begin{aligned}
\frac{\dot{a}}{2 a}= & \frac{\dot{J}_{\mathrm{gr}}}{J_{\mathrm{orb}}}=-8.315 \times 10^{-10}\left(\frac{m_{1}}{M_{\odot}}\right)\left(\frac{m_{2}}{M_{\odot}}\right)\left(\frac{M}{M_{\odot}}\right)\left(\frac{a}{R_{\odot}}\right)^{-4} \\
& \times\left(\frac{1+\frac{7}{8} e^{2}}{\left(1-e^{2}\right)^{5 / 2}}\right) \mathrm{yr}^{-1}
\end{aligned}
$$

$$
\begin{aligned}
\frac{\dot{e}}{e}= & -8.315 \times 10^{-10}\left(\frac{m_{1}}{M_{\odot}}\right)\left(\frac{m_{2}}{M_{\odot}}\right)\left(\frac{M}{M_{\odot}}\right)\left(\frac{a}{R_{\odot}}\right)^{-4} \\
& \times\left(\frac{\frac{19}{6}+\frac{121}{96} e^{2}}{\left(1-e^{2}\right)^{5 / 2}}\right) \mathrm{yr}^{-1} .
\end{aligned}
$$

Gravitational radiation could explain the formation of cataclysmic variables (CVs) with orbital periods less than $3 \mathrm{~h}$, while magnetic braking of the tidally coupled primary by its own magnetic wind would account for orbital angular-momentum loss from CVs with periods up to $10 \mathrm{~h}$ (Faulkner 1971; Zangrilli et al. 1997). We use the formula for the rate of angular-momentum loss due to magnetic braking derived by Rappaport et al. (1983) and Skumanich (1972):

$\dot{J}_{\mathrm{mb}}=-5.83 \times 10^{-16} \frac{m_{\mathrm{env}}}{m}\left(\frac{r \omega_{\text {spin }}}{\mathrm{R}_{\odot} \mathrm{yr}^{-1}}\right)^{3} M_{\odot} R_{\odot}^{2} \mathrm{yr}^{-2}$,

where $r, m_{\mathrm{env}}$ and $m$ are the radius, envelope mass and mass of a star with a convective envelope, and $\omega_{\text {spin }}$ is the spin angular velocity of the star.

\subsubsection{Tidal interaction}

Observations indicate that stellar rotation in a close binary system tends to synchronize with the orbital motion (Levato 1974; Strassmeier 1996). The two tidal dissipation processes, i.e. turbulent dissipation of the equilibrium tide and radiative damping of the dynamical tide, are able to account for the observed orbital circularization of close binary and components rotating in synchronism with orbital motion; see Zahn (2005) for a review. The calculation of tidal interaction in our model follows Hurley et al. (2002) and adopts an equilibrium tide for the stars with a convective envelope (Rasio et al. 1996) and a dynamical tide for the stars with a radiative envelope (Zahn 1975, 1977).

In addition, we note that a hydrodynamical mechanism may account for the orbital circularization as it is concomitant with the hydrodynamical spin-down of the components both in earlytype and late-type detached close binaries (Tassoul 1988). Since 
this mechanism would be more effective than the tidal interaction at circularizing the orbit of a binary, we adopt the tidal dissipation as an upper limit to the circularization timescale.

The tidal synchronisation time is then

$t_{\text {synch }}=1.3 \times 10^{7} q_{2}^{2}\left(\frac{m_{2} / M_{\odot}}{l_{2} / L_{\odot}}\right)^{5 / 7}\left(\frac{r_{2}}{a}\right)^{-6} \mathrm{yr}$

for a tide raised on a white dwarf secondary of mass $m_{2}$ (Campbell 1984), where $r_{2}, l_{2}$ and $q_{2}$ represent its radius, luminosity and mass ratio respectively. $t_{\text {synch }}$ would be a lower limit if tidally-induced non-radial oscillations were included. The orbit of DWDs should be circularized already, and a circularization time-scale is not relevant. However, the synchronization timescale is essential as the companion may be spun up by mass transfer (Hurley et al. 2002).

\subsubsection{Mass transfer in compact binaries}

As a result of gravitational radiation, mass transfer or direct merger between two compact binaries is inevitable if the compact object with lower mass fills its Roche lobe. The analytic mass-radius relation for zero-temperature white dwarfs, is (Nauenberg 1972)

$r=0.01125 R_{\odot}\left(m^{-2 / 3}-m^{\prime 2 / 3}\right)^{1 / 2}$,

where $m^{\prime}=m / 1.433 M_{\odot}$ is the mass in terms of the Chandrasekhar mass. Combining with Eq. (9), we deduce an equilibrium mass transfer rate

$\dot{m}_{2}=\frac{C_{2} L_{\mathrm{Edd}} / \phi_{\mathrm{r} 1}-2 m_{2} / \tau_{\mathrm{GR}}}{\xi_{\mathrm{ad}}-C_{1}-C_{2} \phi_{\mathrm{L} 2} / \phi_{\mathrm{r} 1}}$,

where $L_{\mathrm{Edd}}=4 \pi r_{2}^{2} \mathrm{cg} / \kappa$ is the Eddington luminosity, $\kappa=0.2(1+$ $X) \mathrm{cm}^{2} \mathrm{~g}^{-1}$ is the opacity of the accreted gas, assumed here to be due to electron scattering, $X$ is the hydrogen mass fraction, $g$ is the gravitational acceleration, and other coefficients are as given by Han \& Webbink (1999). $\tau_{\mathrm{GR}}$ is the time scale for orbital angular-momentum loss due to gravitational radiation (Landau \& Lifshitz 1958) with

$\tau_{\mathrm{GR}}=\frac{5}{32} \frac{c^{5}}{G^{3}} \frac{a^{4}}{m_{1} m_{2} M}$.

and $C_{1}, C_{2} \xi_{\text {ad }}$ and $\phi_{\mathrm{L} 1}$ as given by Han \& Webbink (1999).

For stable mass transfer, Han \& Webbink (1999) give the critical mass ratio

$q=\frac{m_{2}}{m_{1}}<q_{\mathrm{c}} \approx 0.7-0.1\left(m_{1} / M_{\odot}\right)$.

Here, $m_{2}$ is donor. If $q \gtrsim q_{\mathrm{c}}$, the mass transfer becomes dynamically unstable, eventually causing a runaway merger.

Nelemans et al. (2001a) give an alternative value $q_{\mathrm{c}} \approx 5 / 6+$ $\zeta\left(m_{2}\right) / 2, \zeta \equiv \mathrm{d} \ln r / \mathrm{d} \ln m$, using the size of the Roche lobe and the mass transfer rate derived by Paczyński (1967). We adopt the Han \& Webbink (1999) approximation.

Finally, we give a formula for the Eddington accretion rate (Cameron \& Mock 1967; Hurley et al. 2002)

$\dot{M}_{\text {Edd }}=2.08 \times 10^{-3}(1+X)^{-1} r_{1} M_{\odot} \mathrm{yr}^{-1}$,

which places a significant limit on the amount of mass accreted by compact objects.

We set this limit only for the accretion of compact objects. However, whether the Eddington limit should be applied or not needs to be further investigated, since the luminosity generated by accretion in excess of this limit might be carried away from the system via a strong wind, or transfered to a disc. SuperEddington accretion rates may be important for the formation of low-mass X-ray binaries and millisecond pulsars (Webbink \& Kalogera 1997) and also for modeling X-ray emission from quasars due to accretion from a disc onto a fast rotating black hole (Beloborodov 1998). Imposing the limit diminishes the birth rate of type Ia supernovae (Livio 2000).

\subsubsection{The formation channels for compact binaries}

One can find a detailed discussion of the formation channels of compact binaries in Han (1998). We here only summarize the three main evolution channels which produce the majority of compact binaries:

I: stable RLOF + CE ejection;

II: CE ejection $+\mathrm{CE}$ ejection;

III: exposed core $+\mathrm{CE}$ ejection.

In channel I, a binary with mass ratio $q=m_{1} / m_{2}$ less than some critical value $q_{\mathrm{c}}$ will experience dynamically stable mass transfer if the primary fills its Roche lobe while the star is in the Hertzsprung gap or on the red giant branch. The primary will become a compact object and the orbital separation will change as

$-\mathrm{d} \ln a=2 \mathrm{~d} \ln m_{2}+2 \alpha_{\mathrm{RLOF}} \mathrm{d} \ln m_{1}+\mathrm{d} \ln \left(m_{1}+m_{2}\right)$

where $\alpha_{\text {RLOF }}$ is the mass-transfer efficiency for stable Rochelobe overflow (RLOF) (Han et al. 1995). Here, we take $\alpha_{\mathrm{RLOF}}=$ 0.5 (Paczyński \& Ziółkowski 1967; Refsdal et al. 1974). Subsequently, if the secondary fills its Roche lobe while it is in the Hertzsprung gap or on the red giant branch, then RLOF will occur.

If the adiabatic response of the radius of the mass donor is less than the change of its Roche lobe radius with respect to a change of mass, i.e. $\left(\frac{\partial \ln R_{\text {donor }}}{\partial \ln M_{\text {donor }}}\right)_{\text {ad }}<\left(\frac{\partial \ln R_{\text {RLOF }}}{\partial \ln M_{\text {donor }}}\right)_{\text {RLOF }}$, mass transfer will be unstable and a common envelope (CE) will form. Interaction (friction) between the compact cores and the $\mathrm{CE}$ will convert orbital energy into kinetic energy, heating and expanding the CE. If the energy conversion mechanism is sufficiently efficient, the CE will be expelled and a compact binary with a short orbital period will result.

Channel II demands that the binary has $q>q_{\mathrm{c}}$ so that, if the primary fills its Roche lobe while in the Hertzsprung gap or on the red giant branch, unstable mass transfer will lead to the formation of a CE. Similarly, if sufficient orbital energy can be extracted, the CE will be ejected to leave a binary containing a compact object. Following evolution of the secondary away from the main sequence, a second CE could form and be ejected to leave a compact binary.

Channel III represents a variation of channels I and II in which the envelope of a massive primary is removed by a stellar wind rather than a first $\mathrm{CE}$ ejection. $\mathrm{CE}$ ejection following evolution of the secondary may also give rise to a compact binary.

In summary, $\mathrm{CE}$ plus $\mathrm{CE}$ represents the major channel. Up to $74 \%$ (Han 1998), or $67 \%$ in our model (see Table 3), of compact binaries may be generated through this channel depending on the choice of parameters. This may also be the most efficient channel to produce compact binaries with very short orbital periods. The stable RLOF plus CE channel can account for up to $50 \%$ of compact binaries (Han 1998), or 30\% in our model. Exposed core plus CE is a minor channel which could make a significant contribution when a binary evolves with a strong tidally-enhanced 
Table 1. Density laws and associated parameters.

\begin{tabular}{lccl}
\hline \hline & Density law & $\begin{array}{c}\text { Constants } \\
(\mathrm{kpc})\end{array}$ & $\begin{array}{c}\rho_{\mathrm{c}} \\
\left(M_{\odot} \mathrm{pc}^{-3}\right)\end{array}$ \\
\hline Bulge & $\mathrm{e}^{-\left(r / r_{0}\right)^{2}}$ & $r_{0}=0.5$ & $\frac{M_{\mathrm{b}}}{4 \pi r_{0}^{3}}=12.73$ \\
Thin disc & $\mathrm{e}^{-R / h_{R}} \operatorname{sech}^{2}\left(-z / h_{z}\right)$ & $h_{R}=2.5$ & $\frac{M_{\mathrm{tn}}}{4 \pi h_{R}^{2} h_{z}}=1.881$ \\
& & $h_{z}=0.352$ & \\
Thick disc & $\mathrm{e}^{-R / h_{R}} \mathrm{e}^{-z / h_{z}^{\prime}}$ & $h_{R}=2.5$ & $\frac{M_{\mathrm{tk}}}{4 \pi h_{R}^{2} h_{z}^{\prime}}=0.0286$ \\
& & $h_{z}^{\prime}=1.158$ & \\
Halo & {$\left[\left(1+\left(\frac{a}{a_{0}}\right)^{2}\right)\right]^{-1}$} & $a_{0}=2.7$ & 0.108 \\
\hline
\end{tabular}

Notes. $r$ is the spherical radius from the center of the Galaxy and $r_{0}$ is bulge scale length; $R$ and $z$ are the natural cylindrical coordinates of the axisymmetric disc, $h_{R}$ is the scale length of the disc, $h_{z}$ is the scale height of the thin disc, $h_{z}^{\prime}$ is the scale height of the thick disc; $a$ is the radius of the halo and $a_{0}$ is a constant; $\rho_{\mathrm{c}}$ is the central mass density.

stellar wind. Both the results of Han (1998) and our own show this channel to be negligible for compact binaries with orbital periods shorter than $70 \mathrm{~h}$.

\subsection{Galactic structure}

A detailed model of the Galaxy is important in order to describe the overall distribution of white dwarf binary systems, including the distance of each from the Sun. It is believed that the current Galaxy mainly comprises the bulge, the thin disc, thick disc and the halo. We summarize our approximation for the Galactic density distribution in Table 1.

Figure 1 shows the distributions of distances to the Earth of compact binaries in the Galaxy and the bulge plus the thin disc. We assume that the position of the sun is $R_{\text {sun }}=8.5 \mathrm{kpc}$ and $z_{\text {sun }}=16.5 \mathrm{pc}$ (Freudenreich 1998).

1. We adopt a normal density distribution for the spherical bulge with a cut-off radius of $3.5 \mathrm{kpc}$ (Nelemans et al. 2004),

$\rho_{\mathrm{b}}(r)=\frac{M_{\mathrm{b}}}{4 \pi r_{0}^{3}} \mathrm{e}^{-\left(r / r_{0}\right)^{2}} M_{\odot} \mathrm{pc}^{-3}$,

where $r$ is the radius from the center of the Galaxy, $r_{0}=$ $0.5 \mathrm{kpc}$ is the bulge scale length, and $M_{\mathrm{b}}$ is the mass of bulge (see Sect. 2.4 find the value). Robin et al. (2003) suggest that the structure of the inner bulge $\left(<1^{\circ}\right.$ from the Galactic center) is not yet well constrained observationally. Consequently we here focus on the outer bulge and make no allowance for any additional contribution to the compact-binary population from the central region.

2. A more complicated function is involved in the spatial density distribution of stars in the disc. Sackett (1997) proposed a double exponential distribution. Phleps et al. (2000) derived three functions for the star density distribution in their model of a thin disc plus thick disc (exponential + exponential, hyperbolic secant + exponential, and squared hyperbolic secant + exponential, respectively) from fits to deep star counts carried out in the Calar Alto Deep Imaging Survey. Robin et al. (2003) created a complicated function to construct the structure of the Galactic disc which is in agreement with Hipparcos results and the observed rotation curve.
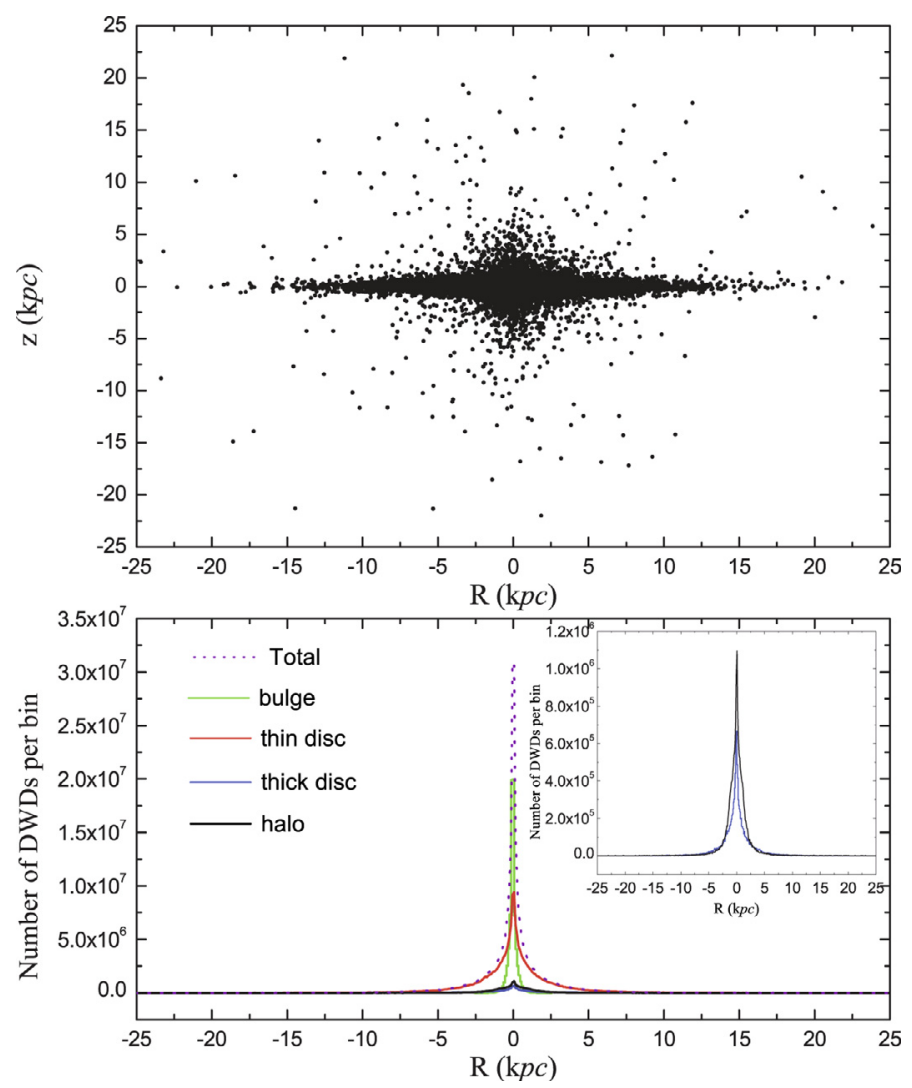

Fig. 1. The top panel shows the distribution of stars in the $R$ (Galactic plane)- $z$ (height) diagram for our Galactic model at age $10 \mathrm{Gyr}$, using a random sample of $\sim 70000$ stars. The lower panel gives the number of DWDs in the Galaxy as a function of galactic radius, using a bin size of $0.1 \mathrm{kpc}$. The inset panel shows the thick disk and halo distribution. We assume there is no immigration between each Galactic component, i.e. no mass transfer, no angular momentum transfer, no collision.

We here model the thin and thick disc components of the Galaxy using a squared hyperbolic secant plus exponential distribution expressed as:

$\rho_{\mathrm{d}}(R, z)=\frac{M_{\mathrm{d}}}{4 \pi h_{R}^{2} h} \mathrm{e}^{-R / h_{R}} \rho(z) M_{\odot} \mathrm{pc}^{-3}$,

where $R$ and $z$ are the natural cylindrical coordinates of the axisymmetric disc, and $h_{R}=2.5 \mathrm{kpc}$ is the scale length of the disc, $h=h_{z}$ for the thin disc, $h=h_{z}^{\prime}$ for the thick disc, and $M_{\mathrm{d}}=M_{\mathrm{tn}}$ is the mass of the thin disc; $M_{\mathrm{d}}=M_{\mathrm{tk}}$ is the mass of the thick disc (see Sect. 2.4 find the values). $\rho(z)$ is the distribution in $z$, with:

$\rho(z)=\operatorname{sech}^{2}\left(-z / h_{z}\right)($ thin disc $)$

and

$\rho(z)=\mathrm{e}^{-z / h_{z}^{\prime}}($ thick disc $)$,

where $h_{z}=0.352 \mathrm{kpc}$ is the scale height of the thin disc and $h_{z}^{\prime}=1.158 \mathrm{kpc}$ is the scale height of the thick disc. We neglect the age and mass dependence of the scale height. 
3. For the halo, we employ a relatively simple density distribution which is consistent with Caldwell \& Ostriker (1981) and Robin et al. $(2003)^{1}$ :

$$
\rho_{\mathrm{h}}(a)=\rho_{\mathrm{c}_{\mathrm{h}}} \times\left(1+\left(\frac{a}{a_{0}}\right)^{2}\right)^{-1},
$$

where $a$ is the radius of the halo, $\rho_{\mathrm{c}_{\mathrm{h}}}=0.108 M_{\odot} \mathrm{pc}^{-3}$ and $a_{0}=2.7 \mathrm{kpc}$.

\subsection{Population synthesis parameters in the Monte Carlo approach}

In order to obtain a sample of compact binaries in the Galaxy, we have performed a Monte Carlo simulation in which we need five physical inputs:

(i) we assume that the star formation rate (SFR) in the bulge and thin disc is the combination of a main star forming process (the first item of the following function) and a minor star formation (the second item of the function),

$$
S F R(t)=11 \mathrm{e}^{-\left(t-t_{0}\right) / \tau}+0.12\left(t-t_{0}\right) M_{\odot} \mathrm{yr}^{-1}, t>t_{0}
$$

where $t$ is time since the halo was formed. Assuming the current age of the Galaxy is $14 \mathrm{Gyr}, t_{0}=4$ Gyr defines the age of the bulge and thin disc to be $10 \mathrm{Gyr}$ and $\tau=9 \mathrm{Gyr}$ yields a current $S F R=4.82 M_{\odot} \mathrm{yr}^{-1}, 1.45 M_{\odot} \mathrm{yr}^{-1}$ in the bulge and $3.37 M_{\odot} \mathrm{yr}^{-1}$ in the thin disc, on average. These values are consistent with Smith et al. (1978), Timmes et al. (1997) and Diehl et al. (2006). We assume that $S F R(t)=0$ in the bulge and thin disc when $0<t<t_{0}$.

In terms of these assumptions, we infer that the combined mass of thin disc and bulge approaches $7.2 \times 10^{10} M_{\odot}$, slightly higher than the mass of $7.0 \times 10^{10} M_{\odot}$ reported by Klypin et al. (2002). Within this mass, the bulge contains $M_{\mathrm{b}}=2.0 \times 10^{10} M_{\odot}$ and the remaining $M_{\mathrm{tn}}=5.2 \times 10^{10} M_{\odot}$ is in the thin disc.

We suppose a burst of star formation, effectively a $\delta$ function, happened at $t=0 \mathrm{Gyr}$ for the halo, and at $t=3 \mathrm{Gyr}$ for the thick disc (Robin et al. 2003) and no star formation thereafter. We assume that the thick disc and the halo attain baryonic masses of $M_{\mathrm{tk}}=2.6 \times 10^{9} M_{\odot}(5 \%$ of thin disc $)$ and $M_{\mathrm{h}}=1.0 \times 10^{9} M_{\odot}$ respectively. These numbers are adopted for simplicity solely to estimate their contribution to the GW signal.

(ii) The initial mass function (IMF) can be constrained by the local luminosity function, stellar density and potential. We here adopted the IMF for the Galactic components based on the results of Robin et al. (2003) and Kroupa et al. (1993) constrained by the observations of Wielen et al. (1983), Popper (1980) and the Hipparcos mission (Creze et al. 1998; Jahreiß \& Wielen 1997).

For the bulge, we suppose an IMF following Robin et al. (2003),

$\xi(m) \propto m^{-2.35}, m>0.7 M_{\odot}$

where $m$ is the primary mass and $\xi(m) \mathrm{d} m$ is the number of stars in the mass interval $m$ to $m+\mathrm{d} m$.

For the thin disc, we adopt the IMF of Kroupa et al. (1993) ${ }^{2}$ which is similar to that of Miller \& Scalo (1979) and

\footnotetext{
1 Although a distribution following $\mathrm{e}^{-a^{1 / 4}}$ or $a^{1 / 4}$ might be a better choice (de Vaucouleurs 1953, 1958), we have chosen the form given here for simplicity.

${ }^{2}$ Kroupa (2001) gives $\xi(m) \propto m^{-2.3}, 0.5<m / M_{\odot}$, see Sect. 3.9.
}

Zoccali et al. (2000). The primary mass is generated using the following formula

$$
\xi(m)= \begin{cases}0.035 m^{-1.3}, & 0.08<m / M_{\odot}<0.5 \\ 0.019 m^{-2.2}, & 0.5<m / M_{\odot}<1.0 \\ 0.019 m^{-2.7}, & 1.0<m / M_{\odot}<100.0\end{cases}
$$

To the thick disc and the halo, we also apply a simple IMF of power law,

$$
\xi(m) \propto m^{-\alpha} .
$$

Here, we take $\alpha=1.5$ for the thick disc and the halo.

We have adopted a metallicity $Z=0.02$ (Population I) for the bulge, thin disc and thick disc, and $Z=0.001$ for the halo. We have also carried out calculations for the thin disc with metallicity $Z=0.001$ in order to see the effect of metallicity on the GW signal.

(iii) We assume a constant mass-ratio distribution (Mazeh et al. 1992; Goldberg \& Mazeh 1994),

$$
n(1 / q)=1,0 \leqslant 1 / q \leqslant 1,
$$

(iv) We employ the distribution of initial orbital separations used by Han (1998) and Han et al. (2003), where they assume that all stars are members of binary systems and that the distribution of separations is constant in $\log a$ ( $a$ is the separation) for wide binaries and falls off smoothly at close separations:

$\operatorname{an}(a)=\left\{\begin{array}{l}\alpha_{\mathrm{sep}}\left(\frac{a}{a_{0}}\right)^{k}, a \leqslant a_{0}, \\ \alpha_{\mathrm{sep}}, a_{0}<a<a_{1} .\end{array}\right.$

where $\alpha_{\text {sep }} \approx 0.070, a_{0}=10 R_{\odot}, a_{1}=5.75 \times 10^{6} R_{\odot}=$ $0.13 \mathrm{pc}, k \approx 1.2$. This distribution implies that the number of binary systems per logarithmic interval is constant. In addition, approximately 50 per cent of all systems are binary stars with orbital periods of less than $100 \mathrm{yr}$.

(v) The distribution of eccentricities of binaries follows $P_{\mathrm{e}}=$ $2 e$ (Nelemans et al. 2001b).

\subsection{Rotation curve and local stellar density}

In order to see the influence of the Galactic model on the rotation curve of the Milky Way, we plotted Fig. 2. We used the Miyamoto-Nagai potential (Miyamoto \& Nagai 1975; Revaz et al. 2009) in cylindrical coordinates for calculating the circular velocity of the bulge and disc components. For the dark matter halo, we adopted the potential of Caldwell \& Ostriker (1981). The observational estimate by Brand \& Blitz (1993) is included for comparison.

From the Galactic model, the total mass of the halo including dark matter is $4.5 \times 10^{11} M_{\odot}$ inside a sphere of radius $50 \mathrm{kpc}$. In this paper, we only focus on the baryonic mass in the halo which is considered to be $1 \times 10^{9} M_{\odot}$. With the SFR adopted here, the baryonic mass in the bulge and disc is at least $2 \times 10^{10} M_{\odot}$ and $5.5 \times 10^{10} M_{\odot}$ respectively, implying that our model requires no dark matter component in the bulge or the thin disc.

Combining the Galactic model and the mass of the Galactic components, the stellar density in the solar neighbourhood is $0.064 M_{\odot} \mathrm{pc}^{-3}$, of which $6.27 \times 10^{-2} M_{\odot} \mathrm{pc}^{-3}$ is in the thin disc, $9.4 \times 10^{-4} M_{\odot} \mathrm{pc}^{-3}$ is in the thick disc, and $2.18 \times 10^{-5} M_{\odot} \mathrm{pc}^{-3}$ is in the halo. This is consistent with the Hipparcos result, $0.076 \pm 0.015 M_{\odot} \mathrm{pc}^{-3}$ (Creze et al. 1998). The local dark matter density in our model is about $0.01 M_{\odot} \mathrm{pc}^{-3}$. 


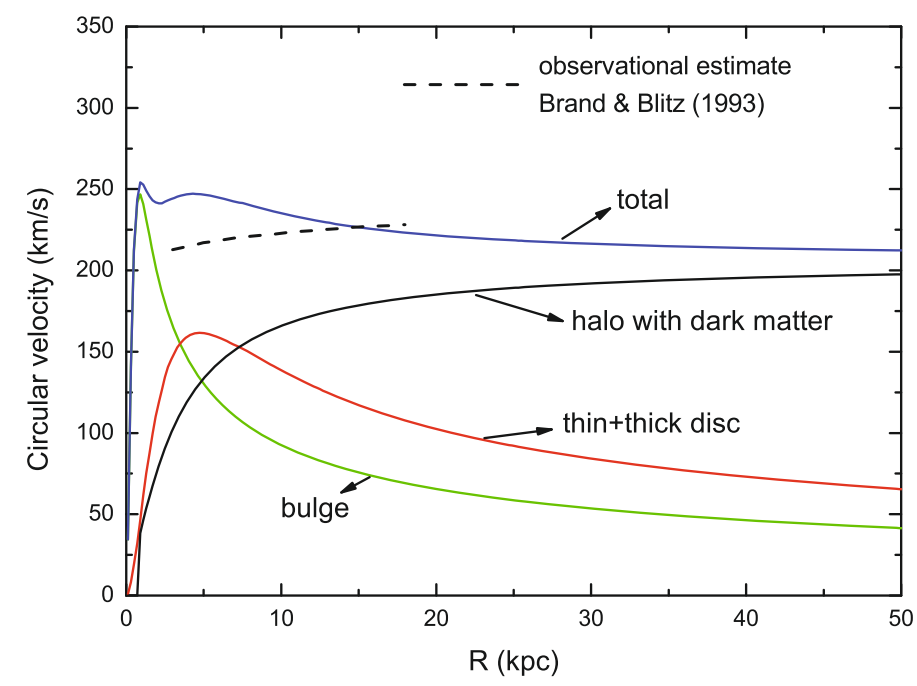

Fig. 2. Circular velocity as a function of galactocentric distance $R$ from the Galatic model, showing the contribution due to different components, i.e. the bulge, thin disc + thick disc, and halo including dark matter. The dashed line indicates the observational estimate by Brand \& Blitz (1993). The spheroidal component due to the interstellar medium was not considered separately.

\subsection{Procedure}

In order to compute birth rates, number densities, space, mass and orbital distribution of DWDs, we have adopted the following procedure. For each Galactic component $g$ (bulge, thin disc, thick disc and halo) having a total mass $M_{g}(t)=\int_{0}^{t} S F R\left(t^{\prime}\right) \mathrm{d} t^{\prime}$ :

1. calculate a sample distribution of $k$ coeval binaries having a total mass $m_{\mathrm{p}}$ and generated by the four Monte-Carlo simulation parameters $m, a, q$ and $e$;

2. follow the evolution of each primordial binary to establish the properties of $n\left(t^{\prime}\right)$ DWDs formed from the original sample, and the number $x\left(t^{\prime}\right)$ of DWDs which merge, where $t^{\prime}$ represents time since formation of each binary;

3. by combining $n\left(t^{\prime}\right)$ and $x\left(t^{\prime}\right)$ with the star formation rate, compute the birth rate of DWDs $v(t)=\int_{0}^{t}\left(n\left(t^{\prime}\right) / m_{\mathrm{p}}\right) \times$ $S F R\left(t-t^{\prime}\right) \mathrm{d} t^{\prime}$

4. compute the merger rate of DWDs $\xi(t)=\int_{0}^{t}\left(x\left(t^{\prime}\right) / m_{\mathrm{p}}\right) \times$ $S F R\left(t-t^{\prime}\right) \mathrm{d} t^{\prime}$

5. evaluate the total number of DWDs $N(t)=\int_{0}^{t} v\left(t^{\prime}\right)-\xi\left(t^{\prime}\right) \mathrm{d} t^{\prime}$;

6. for a given $t$ (e.g. $14 \mathrm{Gyr}$ ), use a second Monte Carlo procedure to generate the orbital properties and spatial distribution of $N(t)$ Galactic DWDs by interpolation and extrapolation on the $n$ DWDs from the simulation;

7. sort the DWDs by orbital frequency;

8. calculate the total strain amplitude $h^{2}$ from the number and distance of DWDs in each frequency bin.

In our population synthesis simulation, we started with $k=$ $1.20 \times 10^{7}$ primordial binaries $\left(4.0 \times 10^{6}\right.$ in the bulge, $5.0 \times 10^{6}$ in the thin disc, $1.0 \times 10^{6}$ in the thick disc and $2.0 \times 10^{6}$ in the halo) yielding a total of $7.66 \times 10^{4} \mathrm{DWDs}$ over all four components of the Galaxy at the present epoch, $t=14$ Gyr.

\section{Results}

\subsection{Birth rates, local densities and numbers of DWDs}

The results of our simulation are shown in Table 2, where we give the birth and merger rates (number per year), local densities $\left(\mathrm{pc}^{-3}\right)$ and total numbers of DWDs. Figures are given for each component of the Galaxy and for the Galaxy as a whole. Table 2 also shows the numbers detectable if we assume that DWDs continue to be detectable up to $10^{8} \mathrm{yr}$ after their formation (Iben et al. 1997). At the end of Table 2, we show the birthrate for type Ia supernovae $=0.0013 \mathrm{yr}^{-1}$, assuming that all SNe Ia are formed from merging double carbon-oxygen (CO) white dwarfs with total mass $>1.378 M_{\odot}$ (Martin et al. 2006).

The local densities of DWDs in our model are $2.1 \times 10^{-4}$, $4.4 \times 10^{-6}$, and $4.1 \times 10^{-7} \mathrm{pc}^{-3}$ in the thin disc, thick disc, and halo, respectively. The predicted density of halo DWDs is significantly less than the observed density of halo white dwarfs $=2 \times 10^{-4} \mathrm{pc}^{-3}$ (Oppenheimer et al. 2001). This could be partially due to the star formation history, as we only have a single star burst of $1 \times 10^{9} M_{\odot}$ at the formation of the halo. If we assume $10 \%$ of halo WDs are in binary systems (Holberg 2009), the observation requires the local density of halo DWDs to be $\approx 2 \times 10^{-5} \mathrm{pc}^{-3}$. This would require the baryonic mass in the halo to be $5 \times 10^{10} M_{\odot}, 11 \%$ of the total mass of the halo, while the dark matter takes up the remaining $89 \%$ of mass of halo.

Table 2 also gives figures for the different thin disc models discussed above. A low metallicity results in a slight increase of the birth rate and number of WD binaries for a given IMF, in line with the results of Han (1998).

Figure 3 shows the distribution of orbital period $P_{\text {orb }}$ as a function of primary mass $\left(m_{1}\right.$, left panel) and mass ratio $(q=$ $m_{2} / m_{1}$, right panel).

\subsection{Chirp masses}

We plot the number density distribution of DWD chirp masses against frequency in Fig. 4. Using Eq. (23) and assuming $15 \mathrm{Gyr}$ for the age of universe, we obtain a relation between chirp mass and a critical frequency for DWDs with a circular orbit, i.e. $e=$ 0.0 ,

$\mathcal{M}=3.05 \times 10^{-7} f_{\mathrm{c}}^{-8 / 5}$.

Figure 4 shows this relation; DWDs with $f>f_{\mathrm{c}}$ will merge within 15 Gyr.

We can understand the distribution of chirp mass from the view of stellar evolution. A star only develops a degenerate helium core to become a helium WD either if its main sequence progenitor evolves to the giant branch and loses its hydrogen envelope prior to core helium ignition, or if the hydrogen-envelope is removed during main-sequence evolution so that the star fails to reach the giant branch and evolves directly from either the main sequence or the Hertzsprung gap to the He WD cooling track. The mass of the He WD will be $\$ 0.5 M_{\odot}$, depending somewhat on metallicity.

If the mass of the He core is $\gtrsim 0.5 M_{\odot}$, core helium burning will be ignited. Following core-helium exhaustion, the star will evolve to become a degenerate carbon-oxygen (CO) or oxygen-neon-magnesium $(\mathrm{ONeMg}) \mathrm{WD}$ with mass in the range of $\approx 0.5-1.44 M_{\odot}$ following an asymptotic giant branch, naked helium giant or hot subdwarf phase, depending on the chemical composition and mass of the progenitor.

With the population synthesis parameters adopted here, Fig. 4 shows a principal ridge in chirp mass at $\mathcal{M} \approx 0.3 M_{\odot}$. Figure 6 demonstrates that this ridge corresponds to the $\mathrm{He}+\mathrm{He}$ DWDs and represents the largest population of DWDs in our model. A smaller and broader ridge is located at $\mathcal{M} \approx 0.65 M_{\odot}$ and corresponds to the CO+CO DWDs (Fig. 6). CO+He DWDs have a range of chirp masses intermediate between the $\mathrm{He}+\mathrm{He}$ 
Table 2. Birth rates, local densities and numbers of DWDs.

\begin{tabular}{|c|c|c|c|c|c|}
\hline & Bulge & Thin disc & Thick disc & Halo & Galaxy \\
\hline$v-\xi$ & $8.0 \times 10^{-3}$ & $\begin{array}{l}{ }^{a} 2.1 \times 10^{-2} \\
{ }^{b} 2.7 \times 10^{-2}\end{array}$ & $1.4 \times 10^{-3}$ & $1.7 \times 10^{-3}$ & $\begin{array}{l}{ }^{a} 3.21 \times 10^{-2} \\
b 3.81 \times 10^{-2}\end{array}$ \\
\hline$N$ & $7.6 \times 10^{7}$ & $\begin{array}{l}{ }^{a} 1.7 \times 10^{8} \\
{ }^{b} 2.3 \times 10^{8}\end{array}$ & $1.2 \times 10^{7}$ & $1.9 \times 10^{7}$ & $\begin{array}{l}{ }^{a} 2.76 \times 10^{8} \\
{ }^{b} 3.37 \times 10^{8}\end{array}$ \\
\hline$N_{\mathrm{o}}$ & $8.0 \times 10^{5}$ & $\begin{array}{l}a 2.1 \times 10^{6} \\
{ }^{b} 2.7 \times 10^{6}\end{array}$ & $1.4 \times 10^{5}$ & $1.7 \times 10^{5}$ & $\begin{array}{l}{ }^{a} 3.21 \times 10^{6} \\
{ }^{b} 3.81 \times 10^{6}\end{array}$ \\
\hline$\xi$ & $4.0 \times 10^{-4}$ & $\begin{array}{l}{ }^{a} 1.4 \times 10^{-3} \\
{ }^{b} 1.5 \times 10^{-3}\end{array}$ & $1.1 \times 10^{-4}$ & $8.3 \times 10^{-4}$ & $\begin{array}{l}{ }^{a} 2.74 \times 10^{-3} \\
{ }^{b} 2.84 \times 10^{-3}\end{array}$ \\
\hline$X$ & $5.4 \times 10^{6}$ & $\begin{array}{l}{ }^{a} 1.1 \times 10^{7} \\
{ }^{b} 1.1 \times 10^{7}\end{array}$ & $1.2 \times 10^{6}$ & $1.2 \times 10^{6}$ & $\begin{array}{l}{ }^{a} 1.88 \times 10^{7} \\
{ }^{b} 1.88 \times 10^{7}\end{array}$ \\
\hline$\rho_{\mathrm{LD}}$ & - & $\begin{array}{l}{ }^{a} 2.1 \times 10^{-4} \\
{ }^{b} 2.8 \times 10^{-4}\end{array}$ & $4.4 \times 10^{-6}$ & $4.1 \times 10^{-7}$ & $\begin{array}{l}{ }^{a} 2.2 \times 10^{-4} \\
{ }^{b} 2.9 \times 10^{-4}\end{array}$ \\
\hline $\begin{array}{l}\mathrm{SNe} \mathrm{Ia} \\
\text { resolved }\end{array}$ & 14800 & $\begin{array}{l}{ }^{a} 18870 \\
{ }^{b} 22670\end{array}$ & & & $\begin{array}{c}v={ }^{a} 1.3 \times 10^{-3} \\
{ }^{a} N_{\mathrm{r}}=33670 \\
{ }^{b} N_{\mathrm{r}}=37470\end{array}$ \\
\hline
\end{tabular}

Notes. $v=$ current birth rate, $\xi=$ current merger rate, $N=$ total number, $N_{\mathrm{o}}=$ the number for DWDs with a detectable component, $X=$ merger number, $\rho_{\mathrm{LD}}=$ local density of DWDs. The last two rows represent the current birth rate of supernovae Ia and the number of resolved DWDs detectable by LISA. Different thin disc models are denoted by ${ }^{(a)} Z=0.02{ }^{(b)} Z=0.001$. Rates $v, \xi$ are in $\mathrm{yr}^{-1}, \rho_{\mathrm{LD}}$ in $\mathrm{pc}^{-3}$.

A DWD is deemed detectable for $10^{8} \mathrm{yr}$ after the second star became a WD. This definition follows Iben et al. (1997) and the values are given for comparison with Han (1998).
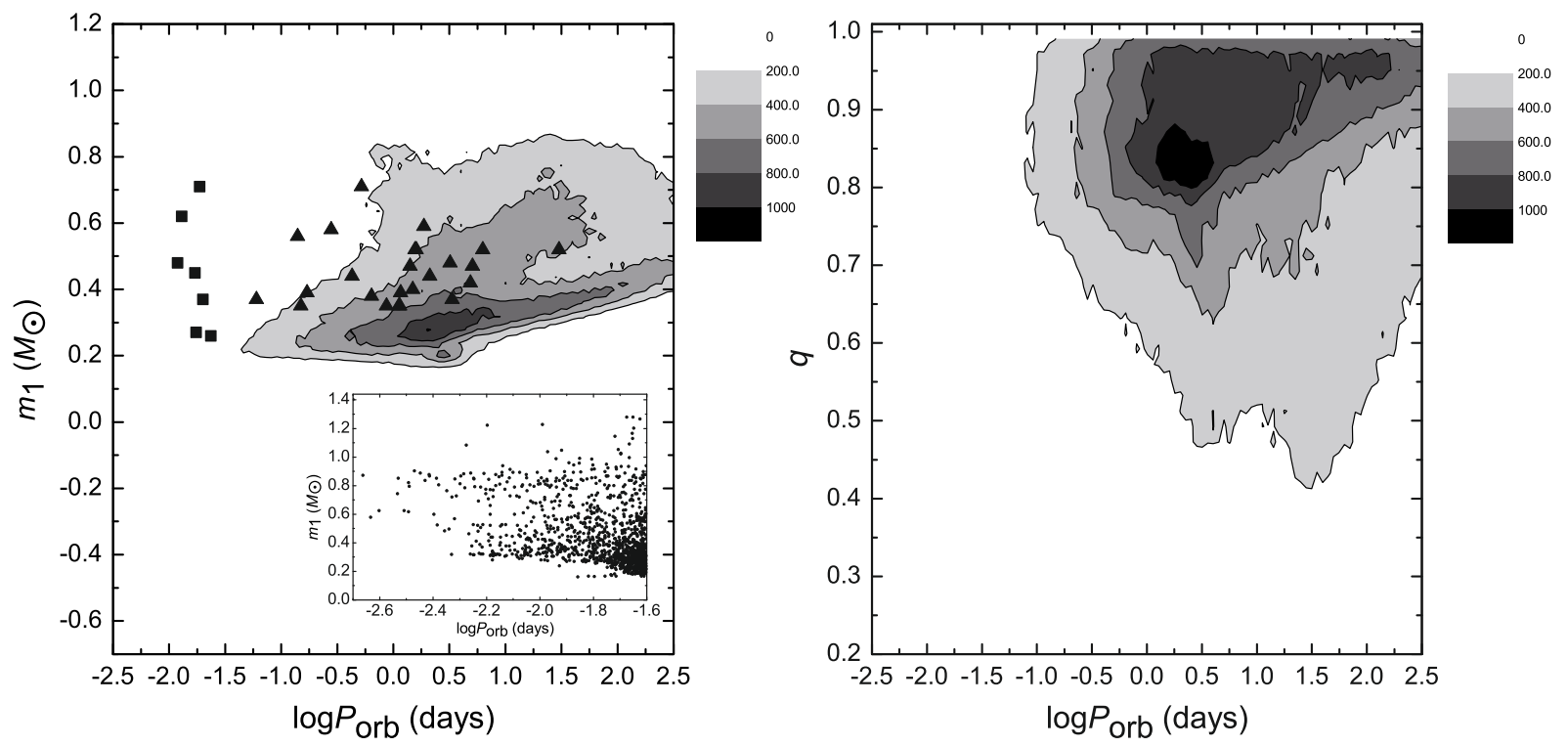

Fig. 3. Distribution of primary masses (left panel), mass ratio $q=m_{2} / m_{1}$ (right panel) and orbital periods of DWDs in our model. The contour scale to the right of the figure represents the number of systems in each bin. The present DWD population is plotted, but the total number of DWDs $\left(2.76 \times 10^{8}\right)$ is reduced by a factor of 100 . Filled triangles and squares are for known WD+WD systems. The inset in the left panel shows the distribution of primary mass and orbital periods at $\log P_{\text {orb }}<-1.6$ days. Bin sizes are $\Delta \log \left(P_{\text {orb }} /\right.$ day $)=0.05, \Delta m_{1} / M_{\odot}=\Delta q=0.02$.

and $\mathrm{CO}+\mathrm{CO}$ DWS (Fig. 6), making the chirp-mass distribution in Fig. 4 appear relatively continuous.

\subsection{The total GW amplitude spectrum from DWDs and comparison with LISA}

In order to synthesize the amplitude spectrum for DWDs, we choose a LISA integration time $T=3.16 \times 10^{7} \mathrm{~s}(1 \mathrm{yr})$, so that the frequency resolution $\Delta f=1 / T=3.17 \times 10^{-8} \mathrm{~Hz}$, which we adopt for the size of the frequency bin. Hereafter, GW frequencies $f$ are given in $\mathrm{Hz}$, unless otherwise stated.

Some frequency bins in the high-frequency domain will contain an individual source if the bin is small enough, arising from a rapid decrease in the number of Galactic binaries towards shorter periods. These are the so-called resolved sources. Evans et al. (1987) discussed the relation between the detector bandwidth $\Delta f$ and integration time $T$. They plotted the amplitude of DWDs with integrations times of $10^{6} \mathrm{~s}$ and $10^{8} \mathrm{~s}$, and found that all binaries with a frequency above $\sim 3 \mathrm{mHz}$ should be resolved if the integration time is $>10^{8} \mathrm{~s}$.

Figure 5 illustrates the predicted total number of DWDs per frequency bin (right panel) and the GW signal (left) these DWDs would produce. Some bins in the range $-3.0<\log f<-1.80$ only contain a single system (Resolved DWDs), making up $\sim 0.012 \%$ (33670, Table 2 ) of the total number of DWDs.

LISA is designed to be a space-based GW detector, consisting of 3 satellites flying in formation to form a Michelson 
S. Yu and C. S. Jeffery: Gravitational waves from double white dwarfs

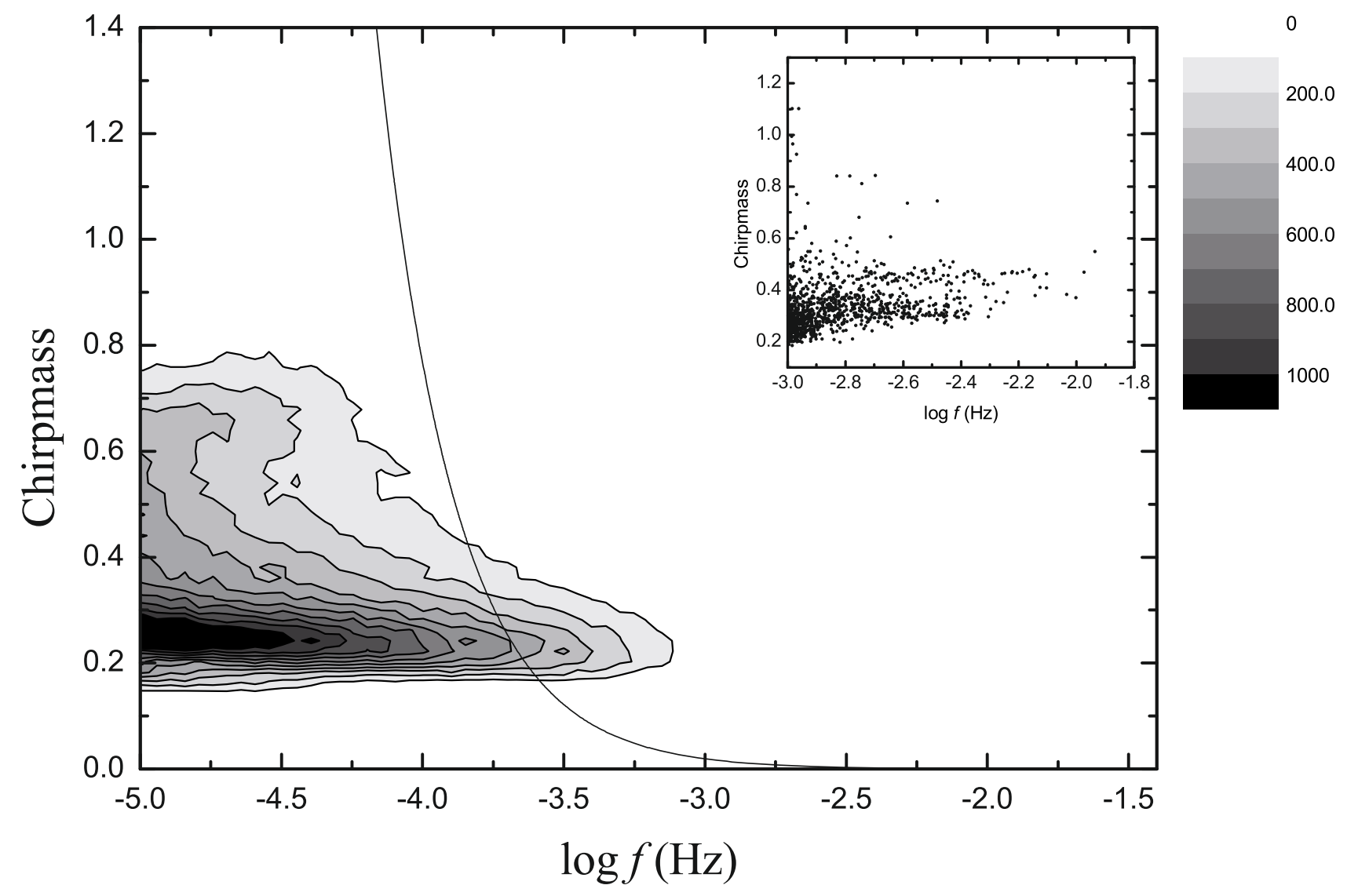

Fig. 4. Distribution of the chirp mass and frequency of DWDs. The contour scale to the right of the figure shows the number of systems in each bin. The present DWD population is plotted, but the total number of DWDs $\left(2.76 \times 10^{8}\right)$ is reduced by a factor of 100 . The solid line denotes the boundary for DWDs which will merge within 15 Gyr. The upper-right panel shows the distribution of chirp mass at high GW frequency $\log f>-3.0 \mathrm{~Hz}$. Bin sizes are $\Delta \log f=0.05$, and $\Delta \mathcal{M}=0.02 M_{\odot}$.

interferometer with an arm length of $5 \times 10^{6} \mathrm{~km}$. Noise arises mainly from the laser tracking system (position noise) and parasitic forces on the proof mass of an accelerometer (acceleration noise) (Larson et al. 2000). We can convert the noise signal to an equivalent $\mathrm{GW}$ signal in frequency space by

$h_{\mathrm{f}}=2 \sqrt{\frac{S_{\mathrm{n}}}{R}}$,

where $S_{\mathrm{n}}$ is the total strain noise spectral density, $h_{\mathrm{f}}$ is the root spectral density and $R$ is the GW transfer function given by Larson et al. (2000).

For a continuous monochromatic source, such as a DWD with a circular orbit, which is observed over a time $T$, the root spectral density will appear in a Fourier spectrum as a single spectral line in the form (Larson et al. 2000)

$h_{\mathrm{f}}=\frac{h}{\sqrt{\Delta f}}=h \sqrt{T}$.

So, for an observation time $T=1 \mathrm{yr}$, the root spectral density $h_{\mathrm{f}}=5.62 \times 10^{3} h$.

To demonstrate the detectability of the predicted GW signals due to DWDs, Figs. 5-9 show the expected LISA sensitivity for $S / N=1$ and 5 .

\subsection{The GW signal from various DWD types and channels}

Figure 6 shows the contribution to the GW signal from different types of DWD in our model, including those containing two helium WDs $(\mathrm{He}+\mathrm{He})$, a carbon-oxygen WD and a helium WD $(\mathrm{CO}+\mathrm{He})$, and two $\mathrm{CO}$ WDs $(\mathrm{CO}+\mathrm{CO})$. DWDs containing at least one $\mathrm{ONeMg}$ white dwarf are designated "ONeMg".

Figure 6 shows that $\mathrm{CO}+\mathrm{CO}$ and $\mathrm{ONeMg}$ DWDs have a stronger total strain amplitude for $\log f<-3.5\left(P_{\text {orb }}>0.9 \mathrm{~h}\right)$, up to almost 0.6 dex higher than that of $\mathrm{He}+\mathrm{He}$ and $\mathrm{CO}+\mathrm{He} \mathrm{DWDs}$, despite being less numerous. Since the strain amplitude for a single DWD of given frequency is proportional to $\mathcal{M}^{5 / 3}$ (Eq. (7)), the larger chirp masses of the $\mathrm{CO}+\mathrm{CO}$ and ONeMg DWDs dominate the numerical superiority of the $\mathrm{He}+\mathrm{He}$ DWDs.

$\mathrm{CO}+\mathrm{CO}$ and $\mathrm{ONeMg}$ DWDs are formed in more massive progenitor binaries than $\mathrm{He}+\mathrm{He}$ and $\mathrm{CO}+\mathrm{He} \mathrm{DWD}$ progenitors. Such binaries undergo RLOF or CE ejection when the stars are physically larger, and hence are in longer period systems and less likely to form short-period DWDs. Consequently the slope of the number-frequency distribution for $\mathrm{CO}+\mathrm{CO}$ and $\mathrm{ONeMg}$ DWDs is steeper than for less-massive DWDs, and the nett contribution to the strain amplitude falls away more quickly.

Consequently, for $\log f>-3.5$, the signals from all four DWD types converge and then at $\log f>-3.0$, the strain amplitude appears to scale roughly as the average chirp mass for the DWD type. The main reason is that the number of systems per frequency bin is here very small and generally $\leq 3$. The contribution from ONeMg DWDs remains highest because of the chirp-mass effect. However, our model predicts negligibly few ONeMg DWDs at $\log f>-2.5$, and negligibly few $\mathrm{CO}+\mathrm{CO}$ DWDs at $\log f>-2.25\left(P_{\text {orb }}<6.65 \mathrm{~min}\right)$, so these contributions vanish at these frequencies. 

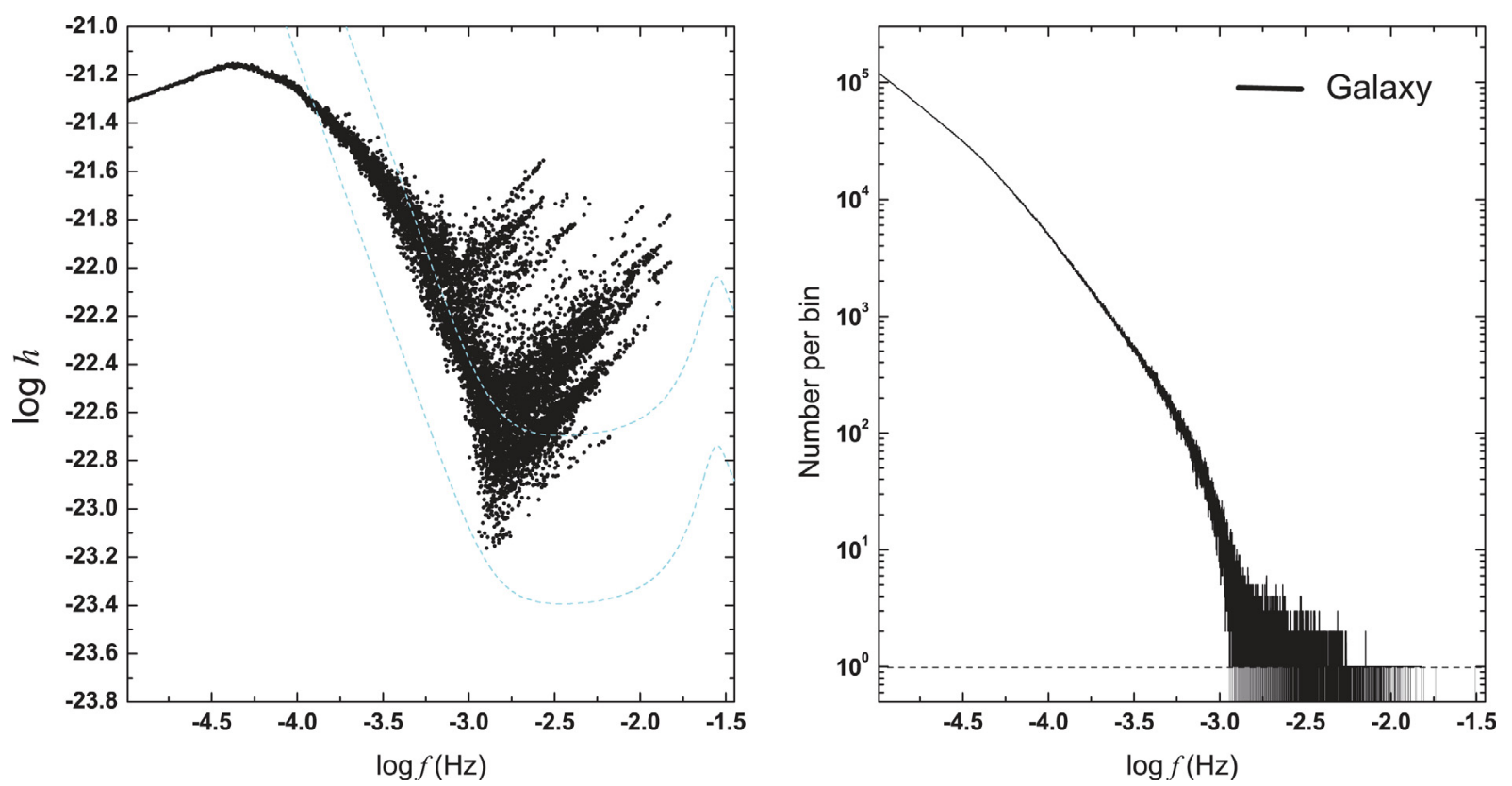

Fig. 5. The number of DWDs per frequency bin (right panel) and their GW signal (left panel). In the left panel, $\log h$ represents the strain amplitude. Colored dashed lines indicate the expected LISA sensitivity for a $S / N=1$ (lower) or 5 (upper). The bin size $\Delta f=3.17 \times 10^{-8} \mathrm{~Hz}$.
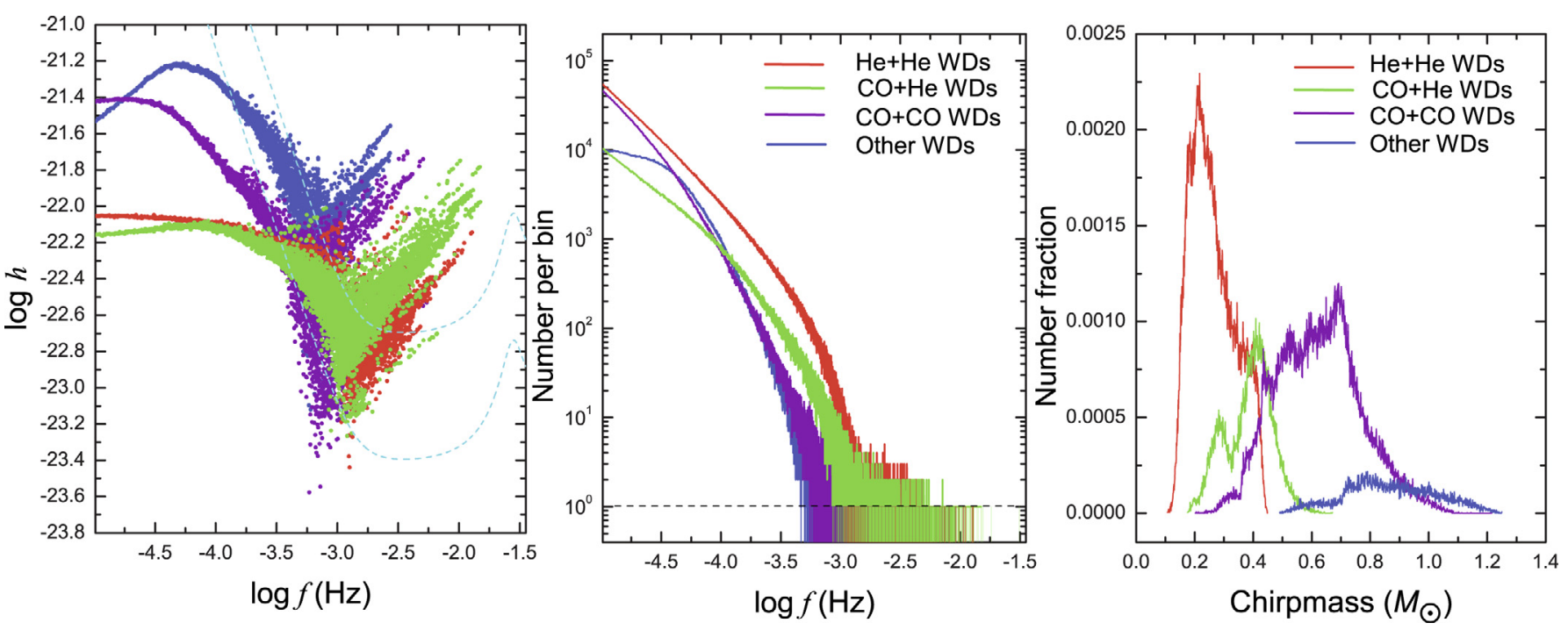

Fig. 6. The GW signal due to different types of DWD. $\log h$ represents the strain amplitude (left panel). The number distribution is shown in the middle panel. The right panel illustrates the distribution of chirp mass due to different DWD types, normalized by the total number $\left(2.76 \times 10^{8}\right)$ of DWDs in the Galaxy (bin size $\Delta \mathcal{M}=0.001 M_{\odot}$ ). The LISA sensitivity and frequency bins are as in Fig. 5 .

Figure 6 shows that $\mathrm{CO}+\mathrm{He}$ DWDs are the dominant GW source at very high frequency $(\log f>-2.3)$. This may be understood as a consequence of evolutionary age. In general, $\mathrm{ONeMg}$ DWDs and CO+CO DWDs correspond to larger progenitor masses and hence a smaller number of progenitors, faster evolution and a lower detection probability. On the other hand, He WDs have larger radii, so $\mathrm{He}+\mathrm{He}$ DWDs tend to merge more quickly after a double $\mathrm{CE}$ phase.

Figure 7 compares the contribution of different DWD formation channels. The stable RLOF+CE and CE+CE channels dominate the production of DWDs, with the $\mathrm{CE}+\mathrm{CE}$ channel generating $66.7 \%$ of all DWDs and dominating the GW signal at all frequencies $\log f>-5$ except in the range $-3.0<\log f<-2.3$ where the contribution from the $\mathrm{RLOF}+\mathrm{CE}$ and $\mathrm{CE}+\mathrm{CE}$ channels may be comparable. The exposed core $+\mathrm{CE}$ channel is a very minor channel $(<3 \%)$ and has almost no influence on the
GW signal at frequencies $\log f>-5\left(P_{\text {orb }}<55.56 \mathrm{~h}\right)$ in our current model, but this would depend on a tidally enhanced stellar wind as discussed by Han (1998) (see also Sect. 2.2.6).

Figure 7 also shows that the highest-frequency DWDs come exclusively from the double CE channel. This channel is expected to be the most important channel for close DWDs because a deep spiral-in phase is necessary to release sufficient orbital energy to eject a common envelope, finally leading to the formation of a very close binary.

In Table 3, we list the total numbers and relative contributions of various types of DWD and evolutionary channels, as well as their current birth rate and the potential number of resolved systems. We find that $\mathrm{CO}+\mathrm{He}$ and $\mathrm{He}+\mathrm{He} \mathrm{DWDs}$ would be the most significant GW sources at $\log f>-2.92\left(P_{\text {orb }}<\right.$ $27.7 \mathrm{~min}$ ), at which frequency resolved sources emerge from our sample. We however cannot neglect the population "ONeMg" 
S. Yu and C. S. Jeffery: Gravitational waves from double white dwarfs
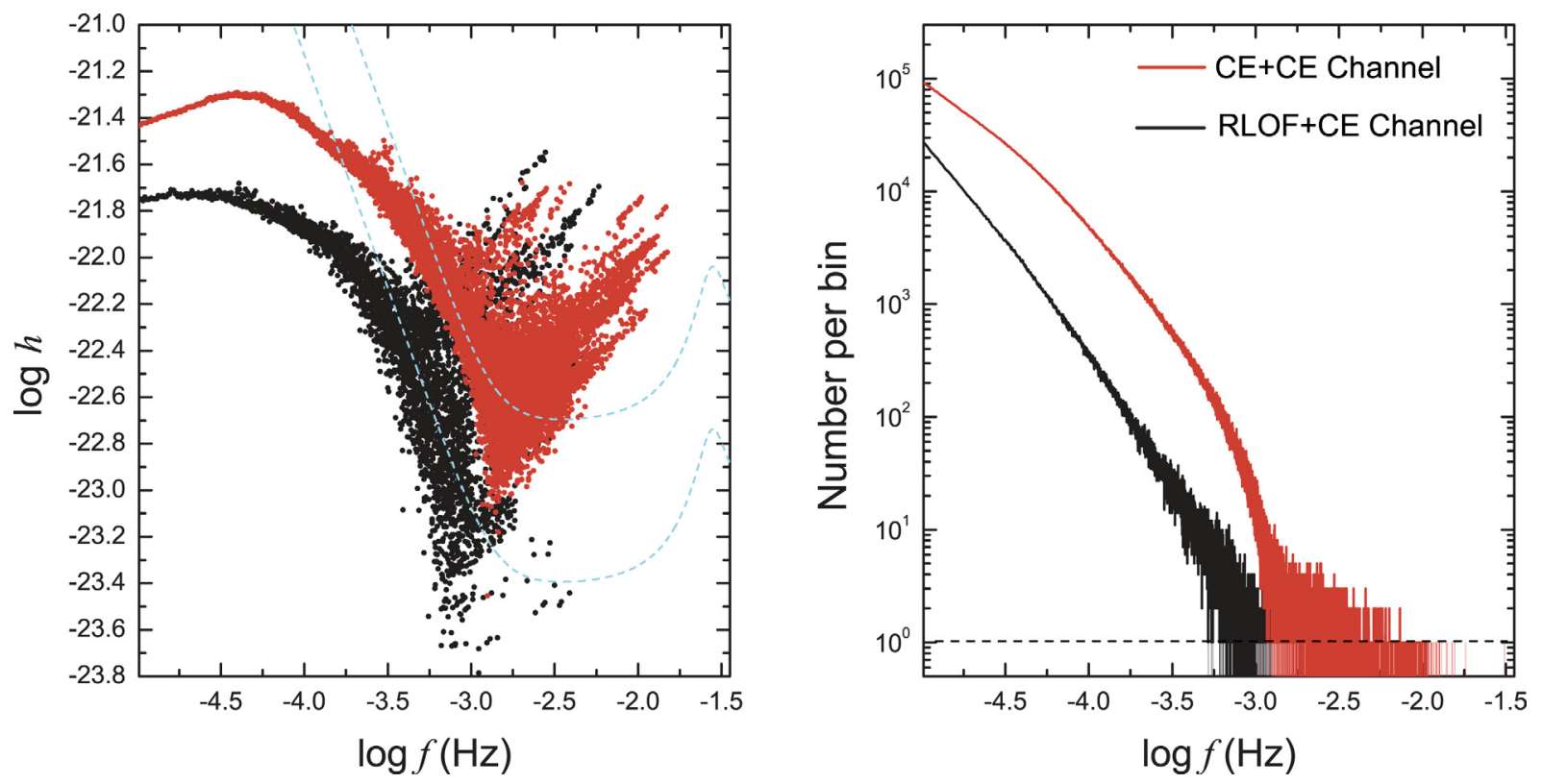

Fig. 7. The GW signal due to DWDs from different formation channels. The left panel presents the strain amplitudes. The number distribution is shown in the right. The LISA sensitivity and frequency bins are as in Fig. 5. Note that the exposed core + CE channel is a very minor channel $(<3 \%)$ and has almost no influence on the GW signal at frequencies $\log f>-5\left(P_{\text {orb }}<55.56 \mathrm{~h}\right)$.

Table 3. Birth rates of DWDs with various types and different formation channels.

\begin{tabular}{lccccc}
\hline \hline & $v$ & $N$ & $N / N_{\text {gal }}$ & $N_{\text {Resolved }}$ & $\%$ \\
\hline types of WD binaries & & & & & \\
He+He & $1.34 \times 10^{-2}$ & $1.06 \times 10^{8}$ & $38.41 \%$ & 19936 & $59.21 \%$ \\
CO+He & $5.07 \times 10^{-3}$ & $4.11 \times 10^{7}$ & $14.89 \%$ & 12852 & $38.17 \%$ \\
CO+CO & $1.15 \times 10^{-2}$ & $1.08 \times 10^{8}$ & $39.13 \%$ & 586 & $1.74 \%$ \\
ONeMg & $2.13 \times 10^{-3}$ & $2.09 \times 10^{7}$ & $7.57 \%$ & 296 & $0.88 \%$ \\
formation channels & & & & & \\
RLOF+CE & $9.86 \times 10^{-3}$ & $8.27 \times 10^{7}$ & $29.96 \%$ & 1061 & $3.15 \%$ \\
CE+CE & $2.12 \times 10^{-2}$ & $1.84 \times 10^{8}$ & $66.67 \%$ & 32609 & $96.85 \%$ \\
other channels & $1.04 \times 10^{-3}$ & $9.3 \times 10^{6}$ & $3.37 \%$ & 0 & 0 \\
Total & $3.21 \times 10^{-2}$ & $2.76 \times 10^{8}$ & & 33670 & \\
\hline
\end{tabular}

Notes. $v=$ current birth rate, $N=$ total number, $\%=$ percentage of the type of DWDs making up the total resolved DWDs in the Galaxy, $N_{\text {Resolved }}=$ number of resolved DWDs. We take the thin disc model with $Z=0.02$.

as they also have a considerable resolved number. $\mathrm{CE}+\mathrm{CE}$ is the dominant formation channel for resolved DWDs, producing 3.8 times more systems than the stable RLOF+CE channel.

\subsection{The GW signal from various Galactic populations}

Figure 8 shows the GW amplitude (right panel) and the number of DWDs per frequency bin (left panel) due to each component of the Galaxy, including bulge, thin disc, thick disc and halo. This figure demonstrates that DWDs in the thin disc and bulge generate a strong strain amplitude which should be observable by LISA. DWDs in the halo and thick disc might make a substantially smaller contribution to the GW amplitude and only at $\log f<-3$. These DWDs will not contribute to the LISA background.

Figure 8 shows that the main difference in the GW signal between different populations is at very high frequencies. At lower GW frequencies the discrepancy is principally caused by the number of DWDs. At very high frequency, the GW signal strongly depends on the star formation history. We note that a persistent quasi-exponential SFR was applied to the bulge and thin disc, but only a single star burst was applied at the beginning of the thick disc and halo. Figure 8 also shows that there is only a slight difference between the signal from the bulge and thin disc, despite having a differnt IMF and spatial distribution.

Figures 1 and 8 show that distance has a only small effect on the DWD signal at high frequency. Therefore, if we assume $d \approx 10 \mathrm{kpc}$, take logarithms of Eq. (8), and combine with the relation between orbital period and GW frequency $\left(f=2 / P_{\text {orb }}\right.$ : circular orbits), we obtain

$\log h=-20.13+\frac{5}{3} \log \left(\frac{\mathcal{M}}{\mathbf{M}_{\odot}}\right)+\frac{2}{3} \log f$.

We plot this relation in Fig. 8 as black dashed lines, which demonstrates the effect of chirp mass on GW amplitude. Thus at high frequency, where individual DWDs can be resolved, chirp mass will strongly influence the strength of the GW signal. It will be used to compare our results with others in Sect. 4. 

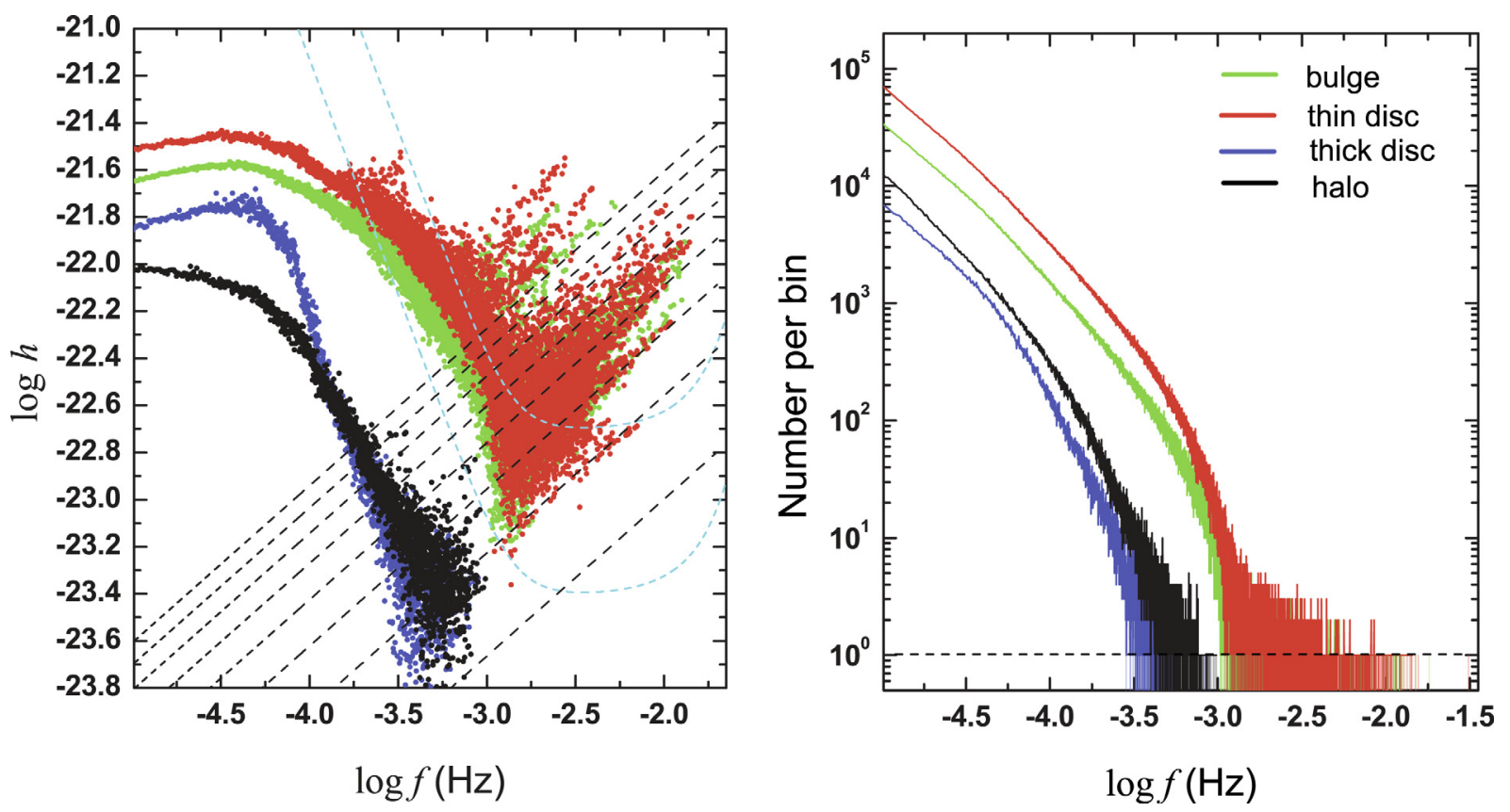

Fig. 8. The GW signal due to DWDs from different populations (see key). The black dashed lines denote the relation of amplitude and frequency from Eq. (41) at chirp masses $0.12,0.22,0.32,0.42,0.52,0.62,0.72$ and $0.82 M_{\odot}$ (from bottom). The LISA sensitivity and frequency bins are as in Fig. 5.

\subsection{The effect of metallicity}

There is a large number of parameters in our model for synthesizing the evolution of large numbers of stars and, since every synthesis takes a large amount of computing time, too many to explore individually in this paper. However, by far the most important parameter in any stellar evolution model, after mass, is metallicity. This will therefore be a crucial factor in determining the properties of the DWD population (Pols et al. 1998; Han 1998; Hurley et al. 2000).

In order to show the influence of metallicity on the GW signal, we performed an additional computation for the thin disc using identical model parameters except for the metallicity. We have compared metallicities $Z=0.02$ and 0.001 .

Figure 9 demonstrates that the shape of the GW spectrum is slightly affected by metallicity at low frequency, but is not much affected at high frequency. A lower metallicity increases both the total number of DWDs, and the number of resolved DWDs. In our thin disc model, these numbers are $1.7 \times 10^{8}\left(2.3 \times 10^{8}\right)$ and 18870 (22 670) for $Z=0.02(0.001)$, respectively.

The difference in the shape of the spectrum arises because at lower metallicities, asymptotic-giant branch stars are able to develop more massive cores, and also achieve higher luminosities and larger radii. This leads to a greater number of more massive WDs in longer initial period orbits, and also to more DWDs surviving.

\subsection{Detached, semi-detached and merged DWDs}

Following formation of a DWD, evolution under the influence of GW may follow one of several paths:

1. if the initial binary is too wide, it will not merge within a Hubble time or equivalent; we adopt 10 Gyr. Considering only the $1.7 \times 10^{8}$ thin disc DWDs (Table 2) this fraction represents some $94 \%$ (or $1.6 \times 10^{8}$ ) of the total;
2. of the remaining $6 \%$ that will evolve into contact within $10 \mathrm{Gyr}$, the majority, $95 \%$ or $9.6 \times 10^{6}$ DWDs are formed with $q>q_{\mathrm{c}}$ and will merge;

3 . the remainder, having $q<q_{\mathrm{c}}$ will undergo stable Roche lobe overflow. This will decrease their mass ratios further, their orbits will widen, and the systems will only stay in contact by further GW radiation. These correspond to some of the AMCVn systems ${ }^{3}$. Their number is $\approx 5 \%$ of those that evolve into contact, or $4.8 \times 10^{5}$ DWDs, or $0.3 \%$ of all DWDs formed in the thin $\operatorname{disc}^{4}$.

\subsection{Resolved sources of GW radiation}

Evans et al. (1987) suggested that degenerate dwarf binaries would be promising sources of GW and pointed out that the detection of GW is related to the integration time. By computing a large number of DWDs, Nelemans et al. (2001b) concluded that wide DWDs dominate the GW signal at $\log f \lessgtr-3.4$, and that AM CVn systems could be resolved at $\log f \gtrsim-2.8$, but would produce a lower signal due to their small number. Subsequently, Nelemans et al. (2004) showed that AM CVn systems would be good candidates for LISA and that $\approx 11000$ AMCVn systems could be resolved. By "resolved", we mean that, over a certain observation time $T$, a frequency bin $(\Delta f=1 / T)$ contains only one binary.

In general, our methods and results are similar. One difference is that, by including their contribution, we confirm that DWDs from the halo and thick disk would make little

\footnotetext{
${ }^{3}$ Nelemans et al. (2001a) also discuss AMCVn systems which are formed from low-mass helium stars with degenerate companions.

4 These figures correspond to model I of Nelemans et al. (2001a), in which there is no tidal coupling between the accretor spin and the orbital angular momentum. Nelemans et al. (2001a) find that with effective tidal coupling, the number of AMCVn systems may increase by two orders of magnitude (their model II). It is not the intention of this paper to discuss AMCVn systems in detail.
} 
S. Yu and C. S. Jeffery: Gravitational waves from double white dwarfs
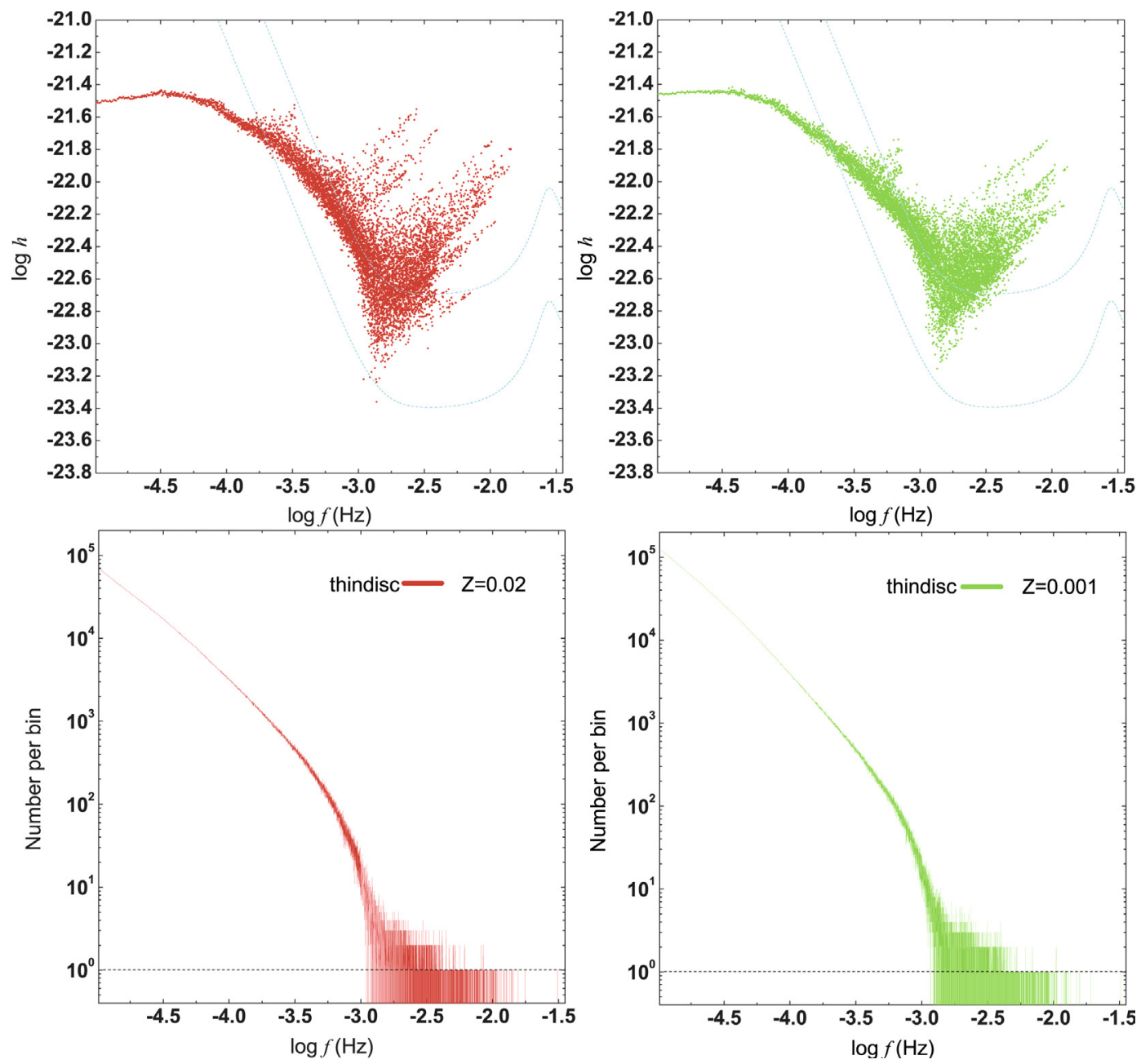

Fig. 9. The GW signal obtained for different thin disc models. The red line is for $Z=0.02$, green for $Z=0.001$. The LISA sensitivity and frequency bins are as in Fig. 5.

contribution to the LISA signal. A second difference is that, by adopting a different IMF, star formation rate and total mass for the bulge and the thin disc, we obtain an increase in the total number of compact DWDs at high frequencies, so that the number of resolved sources increases slightly.

Our results confirm that all DWDs with frequency $\log f>$ -2.25 would be resolved with a LISA integration time equal to 1 yr. In Sect. 3.3, we noted that resolved systems with $\log f>-3.0$ would account for $0.012 \%$ of the total number of DWDs, of which $29 \%$ will become semi-detached. These numbers agree reasonably with previous results, although Nelemans et al. $(2001 \mathrm{~b}, 2004)$ found $\approx 50 \%$ to be semi-detached.

Section 3.4 points out that most resolved DWDs should have formed from the $\mathrm{CE}+\mathrm{CE}$ channel and should be dominated by $\mathrm{CO}+\mathrm{He}$ and $\mathrm{He}+\mathrm{He}$ DWDs. They should be the most significant resolved sources for LISA due to their number and strain amplitude and would dominate the LISA GW signal at $\log f>-2.25$, $\left(P_{\text {orb }}<6.65 \mathrm{~min}\right)$. It may also be possible to infer their type $(\mathrm{CO}+\mathrm{He}$ or $\mathrm{He}+\mathrm{He})$ from their chirp masses (Eq. (42)) by determining $P_{\text {orb }}$ and $\dot{P}_{\text {orb }}$, possibly from optical observations.

$\mathrm{CO}+\mathrm{CO}$ and $\mathrm{ONeMg}$ DWDs have a stronger strain amplitude at $\log f<-3.12\left(P_{\text {orb }}>0.732 \mathrm{~h}\right)$, resulting from their larger chirp masses. For frequencies $-2.25>\log f>-3.12$, the signal from all four types of DWD would overlap.

\subsection{Error analysis}

Modelling stellar populations and hence estimating the numbers, properties and distributions of stars in the Galaxy is subject to several sources of error (or uncertainty). These may naïvely be divided into statistical errors arising from the implementation of the Monte Carlo process, and systematic errors arising from choices made for the model parameters (of which over thirty may be identified in Sect.2; principal values are indicated in Table 4).

The Monte Carlo procedures involve two stages, the first of which creates a sample of $\int n \mathrm{~d} t^{\prime}=1.84 \times 10^{4}$ DWDs in the thin disc for example, on which subsequent samples are interpolated. Assuming Poisson statistics, the error $(1 \sigma)$ in this first number is $\approx \sqrt{\int n \mathrm{~d} t^{\prime}} \approx 1.4 \times 10^{2}$ or $\approx 0.8 \%$. This represents a lower limit on the error in subsequent quantities, including total birth rates, merger rates and numbers of DWDs. The statistical error on the number distributions and strain amplitude spectra will be at least as large as this, increasing as $(N(f) \mathrm{d} f)^{-0.5}$ towards higher frequencies.

In order to investigate some of the systematic errors, we have carried out reduced simulations for the thin disc population with an initial sample of $10^{5}$ binaries and by varying each of the five most important parameters (Table 5). Whilst we omit other parameters from this exploration ( $B$, for example) to save 
Table 4. Main parameters and their value in our simulation.

\begin{tabular}{lccccc}
\hline \hline Stellar evolution parameters & $\gamma$ & $\alpha_{\text {RLOF }}$ & $B$ & $\beta_{\mathrm{w}}$ & $v_{\mathrm{w}}$ \\
& 1.5 & 0.5 & 1000 & 1.5 & 20 \\
& bulge & thin disc & thick disc & halo & \\
\hline age (Gyr) & 10 & 10 & 11 & 14 \\
current $M_{\mathrm{g}}\left(M_{\odot}\right)$ & $2 \times 10^{10}$ & $5.2 \times 10^{10}$ & $2.6 \times 10^{9}$ & $1 \times 10^{9}$ & \\
current SFR $\left(M_{\odot} \mathrm{yr}^{-1}\right)$ & 1.45 & 3.37 & single burst & single burst \\
IMF & $\propto m^{-2.35}$ & Eq. (34) & $\propto m^{-1.5}$ & $\propto m^{-1.5}$ \\
$Z$ & 0.02 & 0.02 & 0.02 & 0.001 \\
$q$ & flat & flat & flat & flat \\
$a$ & Eq. (37) & Eq. (37) & Eq. (37) & Eq. (37) \\
$P_{\mathrm{e}}$ & $\propto 2 e$ & $\propto 2 e$ & $\propto 2 e$ & $\propto 2 e$ \\
\hline
\end{tabular}

Notes. See Table 1 for the Galactic structure parameters. See Sect. 2 for an explanation of the parameters.

Table 5. Estimated errors in DWD birthrates for the thin disk.

\begin{tabular}{lccrr}
\hline \hline$p$ & $\langle p\rangle$ & $\langle\delta p\rangle$ & $\partial v / \partial p$ & $\delta v_{p} / v$ \\
\hline$\gamma$ & 1.5 & 0.1 & $3.4 \times 10^{-4}$ & 0.016 \\
$\log Z$ & -1.7 & 0.5 & $4.3 \times 10^{-3}$ & 0.102 \\
$t \mathrm{Gyr}^{-1}$ & 14 & 1 & $0.1 \times 10^{-3}$ & 0.005 \\
$\alpha_{\mathrm{IMF}}$ & -2.2 & 0.1 & $8.6 \times 10^{-4}$ & 0.041 \\
$a_{\mathrm{SFR}}$ & 11 & 1 & $1.0 \times 10^{-3}$ & 0.047 \\
\hline
\end{tabular}

computation time, their systematics have been discussed by others (Han 1998).

Systematic errors can be estimated by considering the gradient $\partial v / \partial p$ of the birthrate $v$ with respect to a model parameter $p$, and multiplying by an expectation value $\langle\delta p\rangle$ for the uncertainty in $p$. Considering $\gamma$, metallicity (here expressed as $\log Z$ ), age $t$, IMF, and SFR to be representative of inputs with large uncertainty, fractional errors $\delta v_{p} / v \equiv \partial v / \partial p \times\langle\delta p\rangle / v$ are indicated in Table 5. To simplify, we have here assumed the disk IMF takes the simpler form $\xi(m) \approx m^{\alpha}$ and the disk SFR takes the form $a\left(\exp \left(t-t_{0}\right) / \tau\right)+b\left(t-t_{0}\right)$, so that they may be characterised by $\alpha_{\mathrm{IMF}}$ and $a_{\mathrm{SFR}}$ respectively; $\tau$ is constrained by the current SFR. $\left\langle\delta \alpha_{\mathrm{IMF}}\right\rangle \approx 0.1$ is suggested by comparing Kroupa et al. (1993) with Kroupa (2001), while $\left\langle\delta a_{\mathrm{SFR}}\right\rangle \approx 1$ is taken from the uncertainty in the thin disc mass given by Klypin et al. (2002).

The dominant uncertainties in $v$ arise from the metallicity approximation $Z=0.02$, at least in the thin disc, and the SFR. Again making a naïve approximation that all $p$ are independent, adding the error contributions quadratically gives a total relative error $(1 \sigma)$ from these sources $\delta v / v \approx 0.34$.

These numbers are also useful for comparing results between different models by substituting the uncertainty $\delta p$ with the difference between adopted values $\Delta p$.

In our model the Galactic DWD birthrate $v-\xi \approx 3.2 \times$ $10^{-2} \mathrm{yr}^{-1}$ (Table 2). Han (1998) found a similar value $(3 \times$ $\left.10^{-2} \mathrm{yr}^{-1}\right)$. For the thin disc alone we find the DWD birthrate to be $2.1 \times 10^{-2} \mathrm{yr}^{-1}$ while Nelemans et al. (2001b) obtained $2.5 \times 10^{-2} \mathrm{yr}^{-1}$. The slight difference between our model and that of Nelemans et al. (2001b) can be understood primarily in terms of the difference $\Delta a_{\mathrm{SFR}}=3$ and $\Delta \alpha_{\mathrm{IMF}}=0.3$

The birth rate of supernovae Ia $\left(0.0013 \mathrm{yr}^{-1}\right)$ in our model is slightly smaller than that of Han (1998) $\left(0.003 \mathrm{yr}^{-1}\right)$ and Nelemans et al. (2001b) $\left(0.002 \mathrm{yr}^{-1}\right)$, and is probably also a consequence of the IMF and SFR. The fact that we set a limit to the Eddington accretion rate (Eq. (25)) restricts the SN Ia rate (Livio 2000).
Another effect on the birth rate and number arises from tidally-enhanced stellar winds and / or wind-driven mass transfer, which is related to a coefficient $B$ (Sect. 2.2.2). Han (1998) finds that the birth rate and number vary slightly with $B$. We adopt $B=1000$ following Han (1998), but much larger values might be justified; with $B=10000$ (Tout \& Hall 1991), the mass-loss rate could be 150 times larger than the Reimers' rate when the star nearly fills its Roche lobe.

\section{Discussion}

In addition to work by Nelemans et al. (2001b, 2004) already discussed, the question of GW radiation from DWDs and their contribution to the LISA signal has also been addressed by Webbink \& Han (1998), Hils \& Bender (2000), Hiscock et al. (2000), Willems et al. (2007), Liu (2009) and Ruiter et al. (2009), amongst others.

In our model, the GW strain amplitude from the total Galactic DWD population would be $\approx 2.51 \times 10^{-22}-6.31 \times$ $10^{-24} \mathrm{~Hz}^{1 / 2}$ for $\log f>-4.0$, and $\approx 5.01 \times 10^{-22}-5.01 \times$ $10^{-23} \mathrm{~Hz}^{1 / 2}$ for $\log f<-4.0$. The larger strain at lower frequencies is primarily due to the larger number of DWDs per unit frequency.

At $\log f>-3$, the GW signal from DWDs in the bulge and thin disc combined equals the total Galactic GW signal. The thick disc and halo make no contribution (Fig. 8) because all DWDs that formed in these components that will merge have already done so. At lower frequencies $(\log f \leq-3)$, Ruiter et al. (2009) found that the halo signal at $\log f=-3$, for example, $h_{\mathrm{f}}=$ $10^{-20} \mathrm{~Hz}^{1 / 2}\left(\equiv \log h \mathrm{~Hz}^{-1 / 2}=-23.75\right)$ is a factor $\approx 10$ lower than that of the disc+bulge, where $h_{\mathrm{f}}=10^{-19} \mathrm{~Hz}^{1 / 2}\left(\equiv \log h \mathrm{~Hz}^{-1 / 2}=\right.$ $-22.75)$. They estimated there to be $1.5 \times 10^{9}$ halo white dwarfs with a total mass of $10^{9} M_{\odot}$ in a halo with $Z=0.0001$ and age 13 Gyr. At $\log f=-3.3$, we have $\langle\log h\rangle \approx-23.3$, roughly a factor three stronger than Ruiter et al. (2009), but in a halo with $Z=0.001$ and age 14 Gyr.

Another difference between our model and the model of Ruiter et al. (2009) arises from the chirp masses (cf. Sect. 3.5). Figure 8 shows the strain-amplitude relation as a function of chirp masses; since there are only one or two systems in the majority of high frequency bins, it is possible to infer that the chirp masses of most DWDs in our model are greater than $0.1 M_{\odot}$. In comparison, the chirp masses of high-frequency DWDs in the model of Ruiter et al. (2009) appear to be less than $0.1 M_{\odot}$. The different distribution of chirpmass in the two models may be due to differences in $Z$ and age adopted in each case. Our results more closely resemble those of Nelemans et al. (2001b). 
The GW radiation from AM CVn binaries and helium cataclysmic variables was calculated by Hils \& Bender (2000), who found that these two populations only provide a slight enhancement on the confusion noise for LISA below $3 \mathrm{mHz}$ and even no increase at higher frequency. Nelemans et al. (2001b) and Webbink \& Han (1998) showed a confused background GW signal due to DWDs which is similar to ours at low frequency, but different at high frequency.

Hiscock et al. (2000) found that the background signal from halo WD binaries could be five times stronger than the expected contribution from Galactic disk binaries by assuming that the fraction of WDs in binaries is the same in the halo as in the disk. This suggested the possibility of a GW signal from halo DWDs although probably the assumption overestimated the number of DWDs in halo, which would justify further investigation. Willems et al. (2007) considered GW radiation from eccentric DWDs formed through interactions in globular clusters and found LISA could provide unique dynamical identifications of these systems. This requires further investigation.

Since the change of GW frequency (or orbital period) $\dot{f}$ can be measured by electromagnetic observations or LISA, $\mathcal{M}$ is given by

$\dot{f}=5.8 \times 10^{-7}\left(\mathcal{M} / M_{\odot}\right)^{5 / 3} f^{11 / 3} \mathrm{~s}^{-2}$.

Hence, for a small number of high-frequency systems likely to be detected by LISA, it will be possible to determine $\mathcal{M}$ directly. By knowing $\mathcal{M}$ and $P$, we can calculate the distance $d$ from Eq. (7), which is vital for constraining the distribution of DWDs and obtaining a better understanding of the structure of the Galaxy.

In this paper, we have not considered the $\mathrm{BH}$ in the center of the bulge or low-mass BHs elsewhere in the Galaxy. More attention should be paid to these in future work, as they could greatly interfere with the ability of LISA to detect resolved systems (Nelemans et al. 2001b).

\section{Conclusion}

A self-consistent numerical simulation of the gravitational wave signal from the entire population of double white dwarf binaries (DWDs) in the Galaxy has been carried out. We have used a population synthesis approach to follow the stellar evolution, and a comprehensive Galactic model in which we suppose that the Galaxy is composed of a bulge, thin disc, thick disc, and halo. This model demonstrates a significant contribution to the GW signal due to DWDs.

We have discussed the birth rates, local densities and total numbers of DWDs in each fraction of the Galaxy, as well as of supernovae Ia, noting that these are affected by the particular choice of IMF, SFR, metallicity and age adopted in our model (Table 2).

The GW signals from different components of the Galaxy have been computed, as well as the contribution from various types of DWD and from DWDs formed by different formation channels. The corresponding birth rates and numbers have been given (Table 3 ).

Formation channels $\mathrm{CE}+\mathrm{CE}$ and stable RLOF+CE play the most important role to produce DWD populations detectable by LISA at $\log f>-4.5\left(P_{\text {orb }}<17.57 \mathrm{~h}\right)$. DWDs with the shortest orbital periods in our model come from the CE+CE channel. The Exposed Core+CE channel is an almost negligible channel for the formation of DWDs.

We find that $\mathrm{CO}+\mathrm{He}$ and $\mathrm{He}+\mathrm{He}$ white dwarf pairs dominate the GW signal at high frequency $(\log f>-2.3)$, while
$\mathrm{CO}+\mathrm{CO}$ and $\mathrm{ONeMg}$ DWDs make the major contribution at $\log f<-2.3$.

We find that $\approx 33000$ DWDs could be resolved by LISA. Most would have formed through the $\mathrm{CE}+\mathrm{CE}$ channel, and the model favours the majority being $\mathrm{CO}+\mathrm{He}$ and $\mathrm{He}+\mathrm{He}$ DWDs. Ground-based observations might determine which by measuring their chirp masses.

Note added in proof. During the final review of this paper, an article by Ruiter et al. (2010) was published in the Astrophysical Journal. Whilst addressing similar questions to those discussed here, some of the conclusions are different. In particular, for reasons that have yet to be fully understood, the high-frequency $(\log f>-2.8) \mathrm{GW}$ signal in our models is higher.

Acknowledgements. The Armagh Observatory is supported by a grant from the Northern Ireland Dept. of Culture Arts and Leisure. S.Y. thanks the Leverhulme Trust for support. S.Y. and C.S.J. thank the referee for his/her constructive comments and also thank G. Nelemans and Z. Han for the discussion.

\section{References}

Beloborodov, A. M. 1998, MNRAS, 297, 739

Boffin, H. M. J., \& Jorissen, A. 1988, A\&A, 205, 155

Boffin, H. M. J., Cerf, N., \& Paulus, G. 1993, A\&A, 271, 125

Bondi, H., \& Hoyle, F. 1944, MNRAS, 104, 273

Brand, J., \& Blitz, L. 1993, A\&A, 275, 67

Caldwell, J. A. R., \& Ostriker, J. P. 1981, ApJ, 251, 61

Cameron, A. G. W., \& Mock, M. 1967, Nature, 215, 464

Campbell, C. G. 1984, MNRAS, 207, 433

Creze, M., Chereul, E., Bienayme, O., \& Pichon, C. 1998, A\&A, 329, 920

de Vaucouleurs, G. 1953, MNRAS, 113, 134

de Vaucouleurs, G. 1958, ApJ, 128, 465

Diehl, R., Halloin, H., Kretschmer, K., et al. 2006, Nature, 439, 45

Douglass, D. H., \& Braginsky, V. B. 1979, in General Relativity: An Einstein centenary survey, ed. S. W. Hawking, \& W. Israel, 90

Eggleton, P. P. 1983, ApJ, 268, 368

Evans, C. R., Iben, I. J., \& Smarr, L. 1987, ApJ, 323, 129

Farmer, A. J., \& Phinney, E. S. 2003, MNRAS, 346, 1197

Faulkner, J. 1971, ApJ, 170, L99

Freudenreich, H. T. 1998, ApJ, 492, 495

Goldberg, D., \& Mazeh, T. 1994, A\&A, 282, 801

Han, Z. 1998, MNRAS, 296, 1019

Han, Z., \& Webbink, R. F. 1999, A\&A, 349, L17

Han, Z., Podsiadlowski, P., \& Eggleton, P. P. 1995, MNRAS, 272, 800

Han, Z., Podsiadlowski, P., Maxted, P. F. L., \& Marsh, T. R. 2003, MNRAS, 341, 669

Hils, D., \& Bender, P. L. 2000, ApJ, 537, 334

Hils, D., Bender, P. L., \& Webbink, R. F. 1990, ApJ, 360, 75

Hiscock, W. A., Larson, S. L., Routzahn, J. R., \& Kulick, B. 2000, ApJ, 540, L5

Holberg, J. B. 2009, J. Phys. Conf. Ser., 172, 012022

Hurley, J. R., Pols, O. R., \& Tout, C. A. 2000, MNRAS, 315, 543

Hurley, J. R., Tout, C. A., \& Pols, O. R. 2002, MNRAS, 329, 897

Iben, I. J., Tutukov, A. V., \& Yungelson, L. R. 1997, ApJ, 475, 291

Isaacson, R. A. 1968, Phys. Rev., 166, 1272

Jahreiß, H., \& Wielen, R. 1997, in Astronomische Gesellschaft Abstract Series, ed. R. E. Schielicke, 13, 43

Klypin, A., Zhao, H., \& Somerville, R. S. 2002, ApJ, 573, 597

Kroupa, P. 2001, MNRAS, 322, 231

Kroupa, P., Tout, C. A., \& Gilmore, G. 1993, MNRAS, 262, 545

Landau, L. D., \& Lifshitz, E. M. 1958, The classical theory of fields (Oxford: Pergamon Press)

Landau, L. D., \& Lifshitz, E. M. 1975, The classical theory of fields, Course of theoretical physics - Pergamon International Library of Science, Technology, Engineering and Social Studies (Oxford: Pergamon Press), 4th rev. engl. ed.

Larson, S. L., Hiscock, W. A., \& Hellings, R. W. 2000, Phys. Rev. D, 62, 062001 Levato, H. 1974, A\&A, 35, 259

Liu, J. 2009, MNRAS, 400, 1850

Livio, M. 2000, in type Ia Supernovae, Theory and Cosmology, ed. J. C. Niemeyer, \& J. W. Truran, 33

Martin, R. G., Tout, C. A., \& Lesaffre, P. 2006, MNRAS, 373, 263

Mazeh, T., Goldberg, D., Duquennoy, A., \& Mayor, M. 1992, ApJ, 401, 265

Miller, G. E., \& Scalo, J. M. 1979, ApJS, 41, 513

Mironovskii, V. N. 1966, SvA, 9, 752

Miyamoto, M., \& Nagai, R. 1975, Publ. Astron. Soc. Jpn., 27, 533 
Nauenberg, M. 1972, ApJ, 175, 417

Nelemans, G., \& Tout, C. A. 2005, MNRAS, 356, 753

Nelemans, G., Verbunt, F., Yungelson, L. R., \& Portegies Zwart, S. F. 2000, A\&A, 360, 1011

Nelemans, G., Portegies Zwart, S. F., Verbunt, F., \& Yungelson, L. R. 2001a, A\&A, 368, 939

Nelemans, G., Yungelson, L. R., \& Portegies Zwart, S. F. 2001b, A\&A, 375, 890 Nelemans, G., Yungelson, L. R., \& Portegies Zwart, S. F. 2004, MNRAS, 349, 181

Oppenheimer, B. R., Hambly, N. C., Digby, A. P., Hodgkin, S. T., \& Saumon, D. 2001, Science, 292, 698

Paczyński, B. 1967, Acta Astron., 17, 287

Paczyński, B., \& Ziółkowski, J. 1967, Acta Astron., 17, 7

Peters, P. C., \& Mathews, J. 1963, Phys. Rev., 131, 435

Phleps, S., Meisenheimer, K., Fuchs, B., \& Wolf, C. 2000, A\&A, 356, 108

Pols, O. R., Schroder, K., Hurley, J. R., Tout, C. A., \& Eggleton, P. P. 1998, MNRAS, 298, 525

Popper, D. M. 1980, ARA\&A, 18, 115

Press, W. H., \& Thorne, K. S. 1972, ARA\&A, 10, 335

Ramsay, G., Brocksopp, C., Groot, P. J., et al. 2007, in 15th European Workshop on White Dwarfs, ed. R. Napiwotzki, \& M. R. Burleigh, ASP Conf. Ser., 372, 425

Rappaport, S., Verbunt, F., \& Joss, P. C. 1983, ApJ, 275, 713

Rasio, F. A., Tout, C. A., Lubow, S. H., \& Livio, M. 1996, ApJ, 470, 1187

Refsdal, S., Roth, M. L., \& Weigert, A. 1974, A\&A, 36, 113

Reimers, D. 1975, Memoires of the Societe Royale des Sciences de Liege, 8, 369

Revaz, Y., Pfenniger, D., Combes, F., \& Bournaud, F. 2009, A\&A, 501, 171

Robin, A. C., Reylé, C., Derrière, S., \& Picaud, S. 2003, A\&A, 409, 523

Roelofs, G. H. A., Groot, P. J., Benedict, G. F., et al. 2007, ApJ, 666, 1174

Ruiter, A. J., Belczynski, K., Benacquista, M., \& Holley-Bockelmann, K. 2009, ApJ, 693, 383
Ruiter, A.J., Belczynski, K., Benacquista, M., Larson, S.L., \& Williams, G. 2010, ApJ, 717, 1006

Sackett, P. D. 1997, ApJ, 483, 103

Skumanich, A. 1972, ApJ, 171, 565

Smith, L. F., Biermann, P., \& Mezger, P. G. 1978, A\&A, 66, 65

Strassmeier, K. G. 1996, A\&A, 314, 558

Tassoul, J. 1988, ApJ, 324, L71

Timmes, F. X., Diehl, R., \& Hartmann, D. H. 1997, ApJ, 479, 760

Tout, C. A., \& Eggleton, P. P. 1988, ApJ, 334, 357

Tout, C. A., \& Hall, D. S. 1991, MNRAS, 253, 9

Tyson, J. A., \& Giffard, R. P. 1978, ARA\&A, 16, 521

Webbink, R. F. 1984, ApJ, 277, 355

Webbink, R. F. 2008, in ed. E. F. Milone, D. A. Leahy, \& D. W. Hobill, Ap\&SS Library, 352, 233

Webbink, R. F., \& Han, Z. 1998, in Laser Interferometer Space Antenna, Second International LISA Symposium on the Detection and Observation of Gravitational Waves in Space, ed. W. M. Folkner, AIP Conf. Ser., 456, 61

Webbink, R. F., \& Kalogera, V. 1997, in Accretion Phenomena and Related Outflows, ed. D. T. Wickramasinghe, G. V. Bicknell, \& L. Ferrario, ASP Conf. Ser., 121, IAU Colloq., 163, 828

Wielen, R., Jahreiß, H., \& Krüger, R. 1983, in Nearby Stars and the Stellar Luminosity Function, ed. A. G. D. Philip, \& A. R. Upgren, IAU Colloq., 76,163

Willems, B., Kalogera, V., Vecchio, A., et al. 2007, ApJ, 665, L59

Zahn, J. 1975, A\&A, 41, 329

Zahn, J. 1977, A\&A, 57, 383

Zahn, J. 2005, in Tidal Evolution and Oscillations in Binary Stars, ed. A. Claret, A. Giménez, \& J.-P. Zahn, ASP Conf. Ser., 333, 4

Zangrilli, L., Tout, C. A., \& Bianchini, A. 1997, MNRAS, 289, 59

Zoccali, M., Cassisi, S., Frogel, J. A., et al. 2000, ApJ, 530, 418 